\title{
Selbstsubversive Gerechtigkeit: Kontingenz- oder Transzendenzformel des Rechts?*
}

\author{
(Erschienen in: Zeitschrift für Rechtssoziologie 1/2008)
}

\section{I. „Recht und Gesellschaft" ohne Gerechtigkeit}

Die Rechtssoziologie kennt keine Gerechtigkeit. Zwar ermitteln zahlreiche empirische Untersuchungen zur lokalen Gerechtigkeit, was die Leute in unterschiedlichen Konfliktsituationen für gerecht und fair halten. Ebenso zahlreich sind soziologische Theorien über Rechtsnormen, Sanktionen, Professionen und Gerichte. Aber es gibt keine rechtssoziologische Theorie der Gerechtigkeit. ${ }^{1}$ Rechtsund kulturkritische Studien pflegen die Ungerechtigkeiten des Rechts in Bezug auf Geschlechterverhältnisse, auf ethnische Herkunft, auf Güterverteilung und auf kulturelle Lebensbedingungen aufzudecken, aber sie entziehen sich regelmäßig der Frage, was Gerechtigkeit des Rechts positiv bedeuten könnte. Die Normativität der Gerechtigkeit erscheint danach, wenn überhaupt, als ein politisches Projekt und nicht als ein Projekt des Rechts. Ist die Gerechtigkeit selbst - die fundamentale Erwartung der Menschen gegenüber dem Recht - der blinde Fleck der Unterscheidung Recht/Gesellschaft?

Es bedarf erst der externen Beobachter Jacques Derrida und Niklas Luhmann, um diesen blinden Fleck auszuleuchten und die Frage aufzuwerfen: Vermag die Gesellschaftstheorie des Rechts einen gegenüber Moral-, Politik- und Rechtsphilosophie spezifischen Beitrag zu einem heute plausiblen Gerechtigkeitskonzept zu leisten? Autopoiese und Dekonstruktion - in meinen Augen die folgenreichsten Theorieirritationen von „Recht und Gesellschaft" der letzten Jahrzehnte - haben in der Tat das Potenzial, einen solchen Beitrag in zwei verschiedene Richtungen voranzutreiben: Rekonstruktion einer Genealogie der Gerechtigkeit und Beobachtung von Entscheidungsparadoxien des modernen Rechts. ${ }^{2}$ Diese beiden "Stile" pflegt Luhmann, auf sie bezieht sich Derrida: "Der eine Stil ist von begründender und dem Anschein nach ungeschichtlicher Art:

\footnotetext{
* Der Text ist aus den Diskussionen eines 2006 zusammen mit Rudolf Wiethölter veranstalteten Seminars "Dem anderen gerecht werden: Alterität versus Universalität in neueren Theorien der Gerechtigkeit" hervorgegangen. Für kritische Kommentare danke ich besonders Sonja Buckel, Eva Buddeberg, Andreas Fischer-Lescano, Rainer Forst, Malte Gruber, Vaios Karavas, Fatima Kastner, Soo-Hyun Oh, Anton Schütz, und Thomas Vesting.

${ }^{1}$ Roger Cotterell (2006) Law's Community: Legal Theory in Sociological Perspective, Oxford: Clarendon, 2, 60, der mit einer normativ aufgeladenen Sozialtheorie des Rechts dem Thema am nächsten steht, formuliert recht vorsichtig: "Social theory has no direct link with the promotion of justice".

Ihre wichtigsten Texte zum Thema Gerechtigkeit: Jacques Derrida (1999) Préjugés: Vor dem Gesetz, Wien: Passagen; Jacques Derrida (1991) Gesetzeskraft: Der 'mystische Grund der Autorität', Frankfurt: Suhrkamp; Jacques Derrida (1995) Marx' Gespenster: Der verschuldete Staat, die Trauerarbeit und die neue Internationale, Frankfurt: Fischer; Niklas Luhmann (1974) Rechtssystem und Rechtsdogmatik, Stuttgart: Kohlhammer; Niklas Luhmann (1981) "Gerechtigkeit in den Rechtssystemen der modernen Gesellschaft", in: Niklas Luhmann (Hrsg.) Ausdifferenzierung des Rechts: Beiträge zur Rechtssoziologie und Rechtstheorie, Frankfurt: Suhrkamp, 374-418; Niklas Luhmann (1993) Das Recht der Gesellschaft, Frankfurt am Main: Suhrkamp, 214ff.
} 
vorgetragen, vorgeführt werden logisch-formale Paradoxien. Der andere, geschichtlicher und anamnestischer, scheint der eines Lesens von Texten zu sein, einer sorgfältigen Interpretation und eines genealogischen Verfahrens. “3

Im genealogischen Vorgehen erscheint Gerechtigkeit nicht mehr vorrangig als ein Konstrukt des philosophischen Diskurses, sondern ist aus konkreten sozialen Praktiken und ständig sich wandelnden Selbstbildern des Rechts zu rekonstruieren. Hier eröffnen sich Perspektiven auf detaillierte sozialhistorische Analysen mit dem Ziel, die historischen Variationen von Gerechtigkeit und ihre jeweiligen Affinitäten zum Wandel grundlegender Distinktionen in den Sozialstrukturen offen zu legen. ${ }^{4}$ Eine solche Historisierung von Gerechtigkeit gibt in der Tat rechtsphilosophische Ansprüche einer zeitlich und räumlich universell geltenden Gerechtigkeit auf, doch folgt sie nicht zugleich einem Anything-goes-Relativismus. Vielmehr sucht sie die verschütteten Verbindungen von Gerechtigkeitssemantik und Sozialstruktur freizulegen. Und es ist es die besondere Stärke gerade der Rechtssoziologie, mit theoriegeleiteten empirischen Untersuchungen Kovariationen von Gerechtigkeit und Gesellschaftsstrukturen zu analysieren. ${ }^{5}$ Letztlich kann dies in den Versuch ausmünden, ein plausibles Gerechtigkeitskonzept unter heutigen Bedingungen zu reformulieren. Wenn aus sozialtheoretischer Perspektive gezeigt werden kann, dass die Sozialstrukturen von segmentierten und stratifizierten Gesellschaften mit den Semantiken der distributiven und der kommutativen Gerechtigkeit verknüpft gewesen sind, indem sie diese an der Gleichheit der Segmente und der Rangordnung der Sozialhierarchien ausgerichtet haben, ${ }^{6}$ wie könnte dann das Verhältnis zwischen heutigen Gesellschaftsstrukturen und den Semantiken der Gerechtigkeit bestimmt werden? Neben Direktiven für Theorie und Empirie bieten sich normative Impulse für ein verändertes Gerechtigkeitsverständnis im Recht der Gegenwart. Der Re-entry solcher soziologischer Analysen in das Recht öffnete für die Normativität der Gerechtigkeit einen „imaginären Raum“ jenseits von Naturrecht und Positivismus. ${ }^{7}$ An dieser Stelle wird der hochproblematische Hiatus zwischen Rechtsstrukturen und Entscheidungen, der die Paradoxien des Rechts hervortreibt, möglicherweise zu einem tieferen Verständnis von Gerechtigkeit führen - im Sinne von subversiven

Praktiken der Selbst-Transzendierung des Rechts, die in der herrschenden Rechtstheorie und in der Dogmatik nur wenig Beachtung finden. ${ }^{8}$

\section{Statt Reziprozität: Asymmetrie juridischer Gerechtigkeit}

\footnotetext{
${ }^{3}$ Derrida (1991) (Fn. 2), 44.

${ }^{4}$ In je unterschiedlichen Nuancierungen einer solchen „zeitgerechten“ Rechts Reinhart Koselleck (2006) "Begriffsgeschichtliche Probleme der Verfassungsgeschichtssschreibung", in: Reinhart Koselleck (Hrsg.) Begriffsgeschichten: Studien zur Semantk und Pragmatik der politischen und sozialen Sprache, Frankfurt: Suhrkamp, 365-401, 365ff.; Derrida (1991) (Fn. 2), 19; Niklas Luhmann (1981) "Subjektive Rechte: Zum Umbau des Rechtsbewußtseins für die moderne Gesellschaft", in: Niklas Luhmann (Hrsg.) Gesellschaftsstruktur und Semantik Bd. 2, Frankfurt: Suhrkamp, 45-104, 48ff.

${ }^{5}$ In diese Richtung argumentiert der Sammelband von Michael Corsten, Hartmut Rosa und Ralph Schrader (2005.) Die Gerechtigkeit der Gesellschaft, Wiesbaden: Verlag für Sozialwissenschaften.

${ }^{6}$ In dieser Richtung Luhmann (1993) (Fn. 2), 224, 226ff., 233ff.

${ }^{7}$ Zum Zusammenhang von „re-entry“ und „imaginary space“ George Spencer Brown (1997) Gesetze der Form, Lübeck: Bohmeier.

${ }^{8}$ Näher zu den Rechtsparadoxien Gunther Teubner (2003) "Der Umgang mit Rechtsparadoxien: Derrida, Luhmann, Wiethölter", in: Christian Joerges und Gunther Teubner (Hrsg.) Rechtsverfassungsrecht: Recht-Fertigungen zwischen Sozialtheorie und Privatrechtsdogmatik, BadenBaden: Nomos, 25-45, 28ff.
} 
Die Sozialtheorie des Rechts kritisiert die derzeit prominentesten philosophischen Theorien der Gerechtigkeit als nicht historisch genug und nicht soziologisch genug. Auch wenn John Rawls und Jürgen Habermas den Anspruch erheben, das Kantische Gerechtigkeitskonzept unter den gegenwärtigen Bedingungen neu zu formulieren - Rawls integriert Elemente der modernen ökonomischen Theorie, Habermas führt Intersubjektivität und die Evolution normativer Strukturen ein -, weisen ihre Vorstellungen zur Gerechtigkeit doch immer noch die alteuropäische Struktur-Semantik-Relation auf: Universalisierung von Reziprozität, Konsenssuche, Rationalität. ${ }^{9}$ Jeder dieser Gerechtigkeitsaspekte aber muss, nimmt man Derrida und Luhmann beim Wort, durch neue Begrifflichkeiten ersetzt werden: Asymmetrie, Umweltorientierung und das nicht-rationale Andere der Gerechtigkeit.

Rawls und Habermas arbeiten mit dem moralischen Prinzip der Reziprozität zwischen individuellen Akteuren und seiner Universalisierung in generelle und abstrakte Normen, welche die Grundlage für eine gerechte Gesellschaft bilden sollen. Der Schleier des Nichtwissens abstrahiert die Normprojektionen individueller rationaler Akteure von den konkreten Umständen ihres Entstehungszusammenhangs und bringt sie dazu, sich auf faire politische Institutionen zu einigen. In Habermas' idealer Sprechsituation richtet sich an formale Prozeduren die Erwartung, den unverzerrten Ausdruck individueller Interessen ebenso wie deren diskursive Universalisierung in moralisch gerechte Normen garantieren zu können. Doch Polykontexturalität, eine der verwirrendsten Erfahrungen unserer Zeit, lässt grundlegende Zweifel zu, ob diese Varianten des Kantischen Gerechtigkeitskonzepts noch zeitgemäß sind. ${ }^{10}$ Angesichts von Polykontexturalität, also angesichts der Emergenz von hochfragmentierten, intermediären Sozialstrukturen und des Auseinanderdriftens von Interaktionssystemen, formalen Organisationen und Gesellschaftssystem kann man die Gesellschaft nicht mehr von der Interaktion her begreifen. Und ebensowenig kann Gerechtigkeit noch in nachvollziehbarer Weise auf die Universalisierung des Prinzips der Reziprozität zwischen individuellen Akteuren gestützt werden. ${ }^{11}$

Zahlreiche Gesellschaftstheorien haben das problematische Verhältnis von Polykontexturalität und Gerechtigkeit von unterschiedlichen Blickwinkeln her thematisiert. Die Analyse gesellschaftlicher Fragmentierung setzt nicht erst mit den heutigen Theoretikern der Diskurspluralität ein, sondern geht auf Emile Durkheims

\footnotetext{
${ }^{9}$ John Rawls (1975) Eine Theorie der Gerechtigkeit, Frankfurt: Suhrkamp; Jürgen Habermas (1992) Faktizität und Geltung, Frankfurt: Suhrkamp. Ein aufschlussreicher Vergleich ihrer Gerechtigkeitsbegriffe bei Rainer Forst (1999) "Die Rechtfertigung der Gerechtigkeit. Rawls' Politischer Liberalismus und Habermas' Diskurstheorie in der Diskussion", in: Hauke Brunkhorst und Peter Niesen (Hrsg.) Das Recht der Republik. Festschrift für Ingeborg Maus, Frankfurt: Suhrkamp, 105-168.

10 Die abstrakte Formulierung von Polykontexturalität findet sich bei Gotthard Günther (1976) "Cybernetic Ontology and Transjunctional Operations", in: Gotthard Günther (Hrsg.) Beiträge zur Grundlegung einer operationsfähigen Dialektik I, Hamburg: Meiner, 249-283; Gotthard Günther (1976) "Life as Poly-Contexturality", in: Gotthard Günther (Hrsg.) Beiträge zur Grundlegung einer operationsfähigen Dialektik I, Hamburg: Meiner, 283-306.

${ }_{11}$ Zur Kritik am Hochrechnen interaktioneller Reziprozität auf die Gesellschaft Niklas Luhmann (1997) Die Gesellschaft der Gesellschaft, Frankfurt: Suhrkamp, 823ff. Eine konsequente Neuformulierung rechtlicher Reziprozität unter Bedingungen der Polykontexturalität bei Rudolf Wiethölter (1994) "Zur Argumentation im Recht: Entscheidungsfolgen als Rechtsgründe?" in: Gunther Teubner (Hrsg.) Entscheidungsfolgen als Rechtsgründe: Folgenorientiertes Argumentieren in rechtsvergleichender Sicht, Baden-Baden: Nomos, 89-120, 119.
} 
Begriff der organischen Solidarität zurück, auf Max Webers neuen Polytheismus formaler Rationalitäten, auf Wittgensteins Pluralität der Sprachspiele und auf Theodor Adornos soziologische Kritik der Kantischen Moral. ${ }^{12}$ Am deutlichsten analysierte Max Weber die Moderne als je eigenständige Rationalisierung unterschiedlicher Wertesphären und Lebensordnungen, die unlösbare Konflikte zwischen entpersonalisierten Glaubensmächten ausgelöst hat. In einer derartigen Situation kann Gerechtigkeit nicht mehr unter Bezug auf den einen Vernunftgrund, auf Reziprozität und Universalisierung, begründet werden. In Wittgensteins Pluralität der Sprachspiele lassen sich die idiosynkratischen Strukturen der Regeln eines jeden Sprachspiels weder mit Vernunftprinzipien noch mit abstrakten Werten rechtfertigen, sondern einzig und allein auf die Praxis der realen Lebensform zurückführen. Nach Adorno herrscht zwischen einer Universalgerechtigkeit im Kantischen Sinn und der modernen Gesellschaft ein struktureller Widerspruch; ihre Inkommensurabilität mit der vertikalen und der horizontalen Differenzierung der Gesellschaft verwandelt den moralischen Impuls der Gerechtigkeit in sein Gegenteil.

Heute sehen wir uns mit François Lyotards Unterscheidung zwischen litige und différend von hermetisch geschlossenen Diskursen konfrontiert, mit Michel Foucaults Brüchen zwischen inkompatiblen epistémes, mit Niklas Luhmanns Pluralität geschlossener, selbstreferentieller Systeme. ${ }^{13}$ Andere Theorien bewegen sich in der Nähe: Michael Walzers Sphären der Gerechtigkeit oder Nelson Goodmans Weisen der Welterzeugung. ${ }^{14}$ Vor allem rechtspluralistische Theorien und pluralistische Versionen neo-materialistischer Theorien verweisen auf die Beziehung zwischen der gesellschaftlicher Fragmentierung und den unüberwindlichen Differenzen verschiedener Rechtsordnungen in deren Gerechtigkeitsprinzipien. ${ }^{15}$ Ihre Inkompatibilität resultiert aus den Kollisionen realer sozialer Praktiken, welche jeweils eine eigene Rationalität und immer auch eine eigene Normativität entwickeln und damit über ein enormes wechselseitiges Schädigungspotenzial verfügen. In höchster Abstraktion radikalisiert Gotthard Günther Polyzentrizität in die Form einer weitaus bedrohlicheren Polykontexturalität, d.h. in eine Pluralität von sich gegenseitig exkludierenden Perspektiven, die von binären Unterscheidungen konstituiert werden. Diese sind inkompatibel miteinander und können lediglich durch Rejektionswerte überwunden werden, welche ihrerseits zu nichts anderem führen als neuen binären

\footnotetext{
12 Emile Durkheim (1977) Über die Teilung der sozialen Arbeit, Frankfurt: Suhrkamp,152ff.; Max Weber (1968) Gesammelte Aufsätze zur Wissenschaftslehre, Tübingen: Mohr \& Siebeck, 603ff.; Ludwig Wittgenstein (1989) "Philosophische Untersuchungen", in: Ludwig Wittgenstein (Hrsg.) Werkausgabe Bd.1, Frankfurt: Suhrkamp, 234-580, 225ff., 572; Theodor W. Adorno (1973) Negative Dialektik, Frankfurt am Main: Suhrkamp, 294; Theodor W. Adorno (1996) Nachgelassene Schriften Bd. 10: Probleme der Moralphilosophie, Frankfurt am Main: Suhrkamp, 147, 175.

${ }^{13}$ Jean-François Lyotard (1987) Der Widerstreit, 2. Aufl. 1989. München: Fink, 17ff.; Michel Foucault (2002) Die Ordnung der Dinge, Frankfurt am Main: Suhrkamp Kap. 2, 3, 7; für die Wissenschaftsdisziplinen: Michel Foucault (1976) Überwachen und Strafen: Die Geburt des Gefängnisses, Frankfurt: Suhrkamp: für das Recht; Luhmann (Fn. 11), 595ff.

${ }_{14}$ Michael Walzer (1992) Sphären der Gerechtigkeit: Ein Plädoyer für Pluralität und Gleichheit, Frankfurt: Suhrkamp; Nelson Goodman (1990) Weisen der Welterzeugung, Frankfurt: Suhrkamp, $134 f f$.

15 Zum Beispiel Karl-Heinz Ladeur (1992) Postmoderne Rechtstheorie: Selbstreferenz Selbstorganisation - Prozeduralisierung, Berlin: Duncker \& Humblot; Hanne Petersen und Henrik Zahle (1995) Legal Polycentricity: Consequences of Pluralism in Law, Aldershot: Dartmouth; Gunther Teubner (1996) "Altera Pars Audiatur. Das Recht in der Kollision anderer Universalitätsansprüche", 65 Archiv für Rechts- und Sozialphilosophie. Beiheft, 199-220. Ein bemerkenswerter Ansatz der neomaterialistischen Theorie: Sonja Buckel (2007) Subjektivierung und Kohäsion: Zur Rekonstruktion einer materialistischen Theorie des Rechts, Weilerswist: Velbrück, 226ff.
} 
Unterscheidungen. ${ }^{16}$ Die beschriebenen Ansätze stimmen - trotz großer Differenzen in anderen Hinsichten - in einem Punkt überein: dass die Kollision heutiger idiosynkratischer Sinnwelten eine Aussöhnung durch eine sozietale Rationalität, geschweige denn durch eine gesellschaftsübergreifende Gerechtigkeit, ausschließt.

Die Konsequenzen für ein gesellschaftlich adäquates Gerechtigkeitskonzept sind drastisch. Wenn unter heutigen Bedingungen Aristotelische wie auch Kantische Konzepte einer gerechten Gesellschaft ihre Plausibilität verloren haben, können lediglich noch den Fragmenten Gerechtigkeitsattribute zugesprochen werden. Selbst wenn man Rawls oder Habermas auf gegenwärtige Sozialstrukturen übertragen wollte und Reziprozität zwischen Akteuren universalisierte, so müsste man doch mit fragmentierten reziproken Beziehungen beginnen und landete letztlich bei fragmentierten Gerechtigkeiten und nicht bei einer komprehensiven Gerechtigkeit. Wendete man den Schleier des Nichtwissens oder die Bedingungen der idealen Sprechsituation auf einen ökonomischen, vom Effizienzprinzip bestimmten Tausch zweier rationaler Akteure innerhalb eines idealen Marktes an, würde man zwar zu einer universalisierten Gerechtigkeit gelangen, die aber ökonomischer Natur wäre und daher den moralischen, rechtlichen oder politischen Aspekten unseres Zusammenlebens nicht gerecht werden, von ökologischen Fragen ganz zu schweigen. Es ist kein Zufall, dass Rawls sein Gerechtigkeitskonzept auf den Bereich der Politik beschränkt. Er entwickelt sein Modell der Verteilungsprozesse mit Blick auf die institutionalisierte Politik, nicht jedoch für das soziale Gefüge in seiner Gesamtheit. Und sobald er den Versuch unternimmt, jenseits politischer Institutionen auch einen weiteren Kreis sozialer Strukturen zu behandeln, so zeigt sich schnell, dass sein Gesellschaftsmodell der "social union of social unions" aus soziologischer Sicht unhaltbar ist. ${ }^{17}$

Selbst wenn man Gerechtigkeit auf die Gesellschaftsfragmente beschränken wollte, so würde doch die Reziprozitätsbeziehung zwischen individuellen Akteuren als Ausgangspunkt für Gerechtigkeit unter den Bedingungen der Polykontexturalität versagen. Ungerechtigkeiten fragmentierter Institutionen geschehen nicht bloß gegenüber ihren Mitgliedern, was eine Korrektur durch das unter innen herrschende Symmetrieprinzip generalisierter Reziprozitätserwartungen ermöglichte. Gerechtigkeit/Ungerechtigkeit einer fragmentierten Institution ist vielmehr eine asymmetrische Relation, eine Beziehung einer historisch in rekursiven Operationsketten entstandenen institutionalisierten partiellen Rationalität zu ihrer gesellschaftsweiten Öffentlichkeit. Gerechtigkeit müsste dann als eine Supernorm für eine hochentwickelte partielle Rationalität im Rahmen ihrer asymmetrischen Beziehung zu dieser Öffentlichkeit entwickelt werden, nicht aber als eine symmetrische Reziprozitätsbeziehung aus der Interaktion ihrer Mitglieder. In der Sprache der Systemtheorie heißt das: Wenn Gerechtigkeit auf der Reflexivität sozialer Systeme beruht, dann ist die Reflexivität von Interaktionen mit der für sie zentralen Reziprozität weder als Modell für formale Organisationen noch für die großen Funktionssysteme geeignet. Diese benötigen andere Formen der Reflexivität, die sich auf deren interne Logiken stützen, zugleich aber auch ihre internen Logiken übersteigen. Eine auf Gerechtigkeit abzielende Reflexivität müsste dann ganz andere Fähigkeiten entwickeln, nicht die Fähigkeit, reziproke Beziehungen zu universalisieren, sondern die Fähigkeit von Organisationen und Funktionssystemen, die Begrenztheit ihrer spezialisierten Rationalperspektive zu thematisieren und

\footnotetext{
${ }^{16}$ Günther (Fn. 10).

${ }^{17}$ Rawls' vorsoziologisches Gesellschaftskonzept wird besonders deutlich bei John Rawls (1971) A Theory of Justice, Cambridge, Mass.: Harvard University Press, 570ff.
} 
daraus Selbstbeschränkungen bezüglich ihrer expansionistischen Handlungsweisen zu entwerfen. ${ }^{18}$

Eine soziologische Sicht wird also für die Moderne ein Paradigm Lost konstatieren: Gerechtigkeit als das Ideal einer guten Gesellschaft. Doch bedeutet das nicht, dass das Recht, wie es Hans Kelsen vorgeschlagen hat, die Idee der Gerechtigkeit vollends aufgeben müsste. ${ }^{19}$ Vielmehr muss man die alte Idee unter neuen Bedingungen reformulieren und sorgfältig zwischen unterschiedlichen Monokontexturen der Gerechtigkeit differenzieren, zwischen moralischer Gerechtigkeit, politischer Gerechtigkeit, wirtschaftlicher Gerechtigkeit - und besonders juridischer Gerechtigkeit. Die Suche nach einer gerechten Gesellschaft ist heute so wichtig wie seit je, aber für die causa der sozietalen Gerechtigkeit hält die moderne Gesellschaft kein Forum, keine Prozeduren, keine Kriterien bereit. Die Suche nach einer gerechten Gesellschaft kann nicht dem einen Königsweg folgen, sie verzweigt sich bereits von Anfang an in unterschiedliche Pfade. Höchst verschiedene Gerechtigkeitskonzepte werden in spezifischen sozialen Praktiken entwickelt, die jeweils auf ihre Eigenrationalität und Eigennormativität eingestimmt sind. Michael Walzer hat in "Sphären der Gerechtigkeit" in Bezug auf Eigentumsrechte gezeigt, wie unterschiedliche Verteilungsgüter und soziale Kontexte notwendigerweise unterschiedliche Prinzipien der Gerechtigkeit hervorbringen. ${ }^{20}$ Diese Sicht ist zu generalisieren. Politische Gerechtigkeit handelt von Machtakkumulation und vom Konsens über kollektive Entscheidungsfindung, sie gestaltet die grundlegenden Institutionen der politischen Verfassung als eine prekäre Beziehung zwischen Macht-Kompromissen, Interessen-Aggregationen, PolicyErwägungen auf der einen Seite und den externen Ansprüchen der Gesellschaft auf der anderen. Rawls und Habermas leisten sicherlich bedeutende Beiträge zu einer solchen politischen Gerechtigkeit. Doch haben sie erstaunlich wenig zu einer spezifisch juridischen Gerechtigkeit zu sagen, die sich mit der Lösung individueller Konflikte durch eine normgebundene neutrale dritte Instanz auseinandersetzt, mit der angemessenen Ausgestaltung von Gerichtsverfahren, mit der korrekten Anwendung allgemeiner Regeln auf konkrete Fälle und mit den Berücksichtigung der Singularität von Fällen und Personen. So verwundert es nicht, dass Rawls' Justice as Fairness in politischen Kontexten sehr einflussreich war, jedoch im Bereich der Rechtsanwendung nur geringes Echo fand. Wenn judizielle Gerechtigkeit vom Richter verlangt, sich bei der Anwendung genereller Normen zugleich sorgsam auf die Singularitäten des Falls, auf die spezifischen Anliegen der Parteien, auf die Besonderheiten des zugrunde liegenden Sozialkonflikts und auf die beteiligten Personen in ihrer konkreten Unendlichkeit einzulassen, dann ist Rawls' Schleier des Nichtwissens letztlich kontraproduktiv. ${ }^{21}$ Rechtssoziologie muss also ein

\footnotetext{
${ }^{18}$ Dazu Gunther Teubner (2006) "Die anonyme Matrix: Menschenrechtsverletzungen durch „private“ transnationale Akteure", 45 Der Staat, 161-187, 175ff.

${ }^{19}$ Hans Kelsen (1960) "Das Problem der Gerechtigkeit", in: Hans Kelsen (Hrsg.) Reine Rechtslehre, 2. Aufl. Wien: Denticke, 335-444.

${ }^{20}$ Walzer (Fn. 14), 27. Eine ähnliche Kontextualisierung in Bezug auf Gleichheit verfolgt Herlinde Pauer-Studer (2000) Autonom leben: Reflexionen über Gleichheit und Freiheit, Frankfurt am Main: Suhrkamp, 25. Forst unterscheidet vier „Kontexte“ der Gerechtigkeit (Ethik, Recht, Demokratie, Moral), hält aber ihre Integration über „Prinzipien“ der Gerechtigkeit für möglich, Rainer Forst (1994) Kontexte der Gerechtigkeit: Politische Philosophie jenseits von Liberalismus und Kommunitarismus, Frankfurt: Suhrkamp, 388ff., 412.

${ }^{21}$ Sensible Analysen des Konflikts zwischen politischer Gleichheit und individueller Gerechtigkeit bei Christoph Menke (2004) Spiegelungen der Gleichheit, Frankfurt am Main: Suhrkamp, insbesondere $203 f f$.
} 
Gerechtigkeitskonzept entwickeln, das der Eigenrationalität und der Eigennormativität des Rechts Rechnung träg - juridische Gerechtigkeit. Das bedeutet freilich nicht, dass das Recht ein Monopol auf Gerechtigkeit besitzt. Vielmehr koexistieren in verschiedenen Gesellschaftskontexten verschiedene Gerechtigkeitskonzepte, die sich nicht einem einheitlichem Prinzip fügen.

Gleichheit, wie sie von Habermas und von Rawls als konzeptuelle Basis der Gerechtigkeit in Anspruch genommen wird, hat in Recht und in Politik jeweils grundlegend voneinander abweichende Bedeutungen. Politische Gleichheit ergibt sich aus einer Generalisierung, die eine aggregierte Gleichbehandlung der Bürger erfordert, juridische Gleichheit folgt demgegenüber aus einem Individualisierungsprozess, der nach einer Gleich-oder-Ungleich-Behandlung des neuen Sachverhaltes mit alten Fällen fragt. Worin genau unterscheidet sich das dezentral erzeugte Ordnungsmuster juridischer Gleichheit von ethischer Generalisierung und von politischer Aggregation?

Im ersten Zugriff kann man die rekursive Anwendung von rechtlichen Operationen auf die Resultate rechtlicher Operationen in einer Vielzahl von Gerichtsprozessen als denjenigen Vorgang verstehen, der das artifizielle Netzwerk von Rechtsnormen, dogmatischen Begriffen und Prinzipien dezentral erzeugt - und aus dieser „lokalen“ Sicht juridische Konzepte der Gerechtigkeit ausformt. Die permanenten Unterscheidungspraktiken der Gleich-oder-ungleich-Behandlung von Einzelfällen sind ein Mechanismus, der die Rechtsgleichheit deutlich von der auf bindenden Kollektiventscheidungen zielende politischen Gleichheit unterscheidet. Gleiches gleich und Ungleiches ungleich zu behandeln, ist der Auslöser einer iterativen Reihe von Unterscheidungen. Dieser generative Mechanismus oder, wie von Foerster es nennen würde, diese "historische Maschine" erhöht unablässig die Komplexität der rechtlichen Konstrukte. ${ }^{22}$ Weniger interessant sind hierbei die Bindung an Präjudizien, das „stare decisis“, und die Gleichbehandlung gleicher Fälle. Vielmehr sind es die Abweichungen vom Hergebrachten, das "distinguishing" und "overruling“, also gerade die Ungleichbehandlung ungleicher Fälle, welche die Suche nach neuen Rechtskonstruktionen und nach einer spezifisch juridischen Gerechtigkeit erzwingt.

Freilich erfasst man das Problem nur zur Hälfte, wenn man die juridische Gerechtigkeit nur zu gerichtlichen Verfahren in Beziehung setzt, also zur sebstreferentiellen Anwendung vergangener Entscheidungen und Regeln auf neue tatsächlich Situationen. Die andere Hälfte betrifft die permanenten Irritationen des Rechts, die von externen sozialen Prozessen ausgehen und die die juridische Gerechtigkeitssemantik in andere Bahnen lenken. An dieser Stelle tritt die typische Inkongruenz von Rechtsnormen und Dogmatik mit dem Einzelfallkonflikt zutage, der ihrer Kovariation mit der Veränderung entfernter Sozialstrukturen entstammt. ${ }^{23}$ Das Netzwerk rechtlicher Operationen, das auf externe Irritationen reagiert, bildet sich in anderen Kontexten als die Irritationen von Einzelkonflikten, die vor die Gerichte gelangen. Diese externen Irritationen lösen eine eigenständige Dynamik aus, die das Recht in eine unvermeidliche Inkongruenz zu Individualkonflikten, zu den rechtlichen Maßstäben für deren Lösung und zu Prinzipien der Gerechtigkeit hineintreibt. Maschinerien der Produktion sozialer Normen dringen von der Peripherie in die Zentren des Rechts ein, indem sie sozialen Normen in Rechtsnormen transformieren.

\footnotetext{
${ }^{22}$ Heinz von Foerster (1993) Wissen und Gewissen, Frankfurt: Suhrkamp, 350ff., $356 f f$.

${ }^{23} \mathrm{Zu}$ externen Irritationen des Rechts Gunther Teubner (1989) Recht als autopoietisches System, Frankfurt: Suhrkamp, 61ff., 81ff.; Luhmann (1993) (Fn. 2), 550ff.
} 
Die produktivsten außerrechtlichen Normerzeugungsmechanismen sind in formalen Organisationen, in informalen Netzwerken und in Prozessen der Standardisierung und Normalisierung institutionalisiert, die heute mit der Gesetzgebungsmaschinerie und dem vertraglichen Tauschmechanismus konkurrieren ${ }^{24}$. Die Suche nach juridischer Gerechtigkeit kann diese extern produzierten Normen nicht ohne Weiteres zurückweisen, etwa weil sie den Besonderheiten des Individualkonfliktes unangemessen wären. Im Gegenteil, sie extrahiert durch deren juristische Rekonstruktion die Maßstäbe, von denen die Lösung des Einzelkonfliktes erwartet werden kann, und überprüft sie gleichzeitig im Licht des rechtlichen ordre public. Daraus entwickeln sich Schritt für Schritt neue substantielle Gerechtigkeitsaspekte.

Auf diese Weise wandeln sich die Prinzipien der juridischen Gerechtigkeit permanent im Feld ihrer rekursiven Konfrontation mit den beiden genannten Dynamiken, der Entscheidung von Individualkonflikten und der Rezeption sozialer Normen. Das setzt die Semantik der juridischen Gerechtigkeit auf ein anderes Gleis als die politische, moralische oder ökonomische Gerechtigkeit, die jeweils ihren idiosynkratischen Universalisierungen folgen. Zur modernen Erfahrung gehört nicht nur deren Differenz, sondern deren Widerstreit. Gesetzgebung, die von Überlegungen der politischen Gerechtigkeit beherrscht wird, unterminiert die juridische Gerechtigkeit der Gerichtsverfahren. Gleiches gilt für den umgekehrten Fall. Ebenso bewegen sich die Prinzipien moralischer Gerechtigkeit, die auf der Basis wechselseitiger Achtung entwickelt und von der philosophischen Ethik systematisiert worden sind, in einem vergleichbaren Verhältnis des Widerspruchs und der Kritik gegenüber den Forderungen der juridischen Gerechtigkeit.

\section{Statt Konsenstheorien: Ökologische Gerechtigkeit}

Unter den Bedingungen der Polykontexturalität bietet Niklas Luhmann ein soziologisches Konzept: Gerechtigkeit ist die Kontingenzformel des Rechtssystems. ${ }^{25}$ Der Begriff ist schwierig und wird leicht missverstanden. Gemeint ist, dass die Thematisierung von Gerechtigkeit überall im Rechtssystem eine irritierende soziale Dynamik in Gang setzt, die die Kontingenz des Rechts allen drastisch vor Augen führt: Gerechtes Recht könnte/müsste anders sein! Die Gerechtigkeitsirritation beginnt schon beim Auftauchen sozialer Konflikte, setzt sich bei deren Übersetzung in die artifizielle Sprache des Rechts, in der Rechtsanwendungspraxis, in den Anwaltstaktiken, in den Auslegungsstreitigkeiten, in der juristischen Entscheidungsfindung, in der Rechtsdurchsetzung, in der Regelbefolgung fort und endet bei der Nichtbefolgung von Rechtsnormen und entscheidungen, beim Protest der Menschen und ihren Revolten gegen die Ungerechtigkeiten des Rechts. Wie wirkt Gerechtigkeit in diesen Praktiken? Nicht als Regel, nicht als Prinzip, nicht als Wert, und nicht als Entscheidungskriterium des Rechts. Aber auch nicht als externes Kriterium auf das Recht, anhand dessen man die rechtlichen Entscheidungen bemessen könnte, nicht als moralische Tugend, nicht als politisches Ziel, nicht als eine regulative Idee, die man dem positiven Recht entgegenhalten könnte. Diese könnten allesamt entweder gegen andere interne Regeln, Prinzipien, Werte, Kriterien oder gegen andere externe Tugenden, Ziele und Ideen abgewogen werden. Doch im Recht ist Gerechtigkeit keiner Abwägung

\footnotetext{
${ }^{24}$ Teubner (Fn. 15), 200ff.

${ }^{25}$ Luhmann (1993) (Fn. 2), 218ff.; cf. Ralf Dreier (2002) "Niklas Luhmanns Rechtsbegriff", 88 Archiv für Rechts- und Sozialphilosophie, 305-322, 315ff.
} 
zugänglich. In anderen Kontexten ist Gerechtigkeit ein Wert unter vielen, im Recht ist sie die eine Kontingenzformel. Juridische Gerechtigkeit wird als die zentrale, die eine unbestreitbare Orientierungsformel in Anspruch genommen und kann zu keinem inner- oder außerrechtlichen Prinzip in Konkurrenz treten. Als Kontingenzformel des Rechts hat Gerechtigkeit im Recht einen ähnlichen Status wie inn andere Kontingenzformeln auf anderen Gebieten ebenfalls haben: Legitimität in der Politik, Gott in der Religion, Güterknappheit in der Ökonomie, Bildung in der Pädagogik, Limitationalität in der Wissenschaft. ${ }^{26}$ Kontingenzformel heißt: Negationsverbot, Kanonisierung, Unbestreitbarkeit. Und ihre Dynamik enthüllt ein Paradox. Die notwendige Suche nach dem Unbestreitbaren erzeugt, wenn sie als Suche beobachtet werden kann, immer wieder neue Kontingenzen. Notwendige Kontingenz - kontingente Notwendigkeit.

Die Kontingenzformel des Rechts - Gerechtigkeit - erscheint somit als ein notwendiges "Schema der Suche nach Gründen oder Werten, die nur in der Form von Programmen Rechtsgeltung gewinnen können“. ${ }^{27}$ Es handelt sich nicht um ein rechtsinternes oder -externes Prinzip, sondern um eine Selbstbeobachtung der Einheit des Rechts auf der Basis seiner Programme, um eine rechtliche Selbstkontrolle, die mittels der oben erwähnten „historischen Maschine“ des Rechts in den endlosen Praktiken der Gleich- und Ungleichbehandlungen stattfindet. Dies führt Luhmann zu der Definition von Gerechtigkeit: „adäquate Komplexität des konsistenten Entscheidens" 28

In der daran anschließenden rechtssoziologischen Diskussion begegnete man dieser Definition mit äußerster Skepsis. ${ }^{29}$ Wenn Gerechtigkeit nicht dazu imstande ist, substantielle Maßstäbe für individuelle Entscheidungen bereit zu stellen, wenn sie nicht mit einem rechtlichen Wert oder Prinzip zu identifizieren ist und wenn sie auch keine externen ethischen oder politischen Maximen angibt, dann ist sie eine rein formale Gerechtigkeit, die auf die einfache Forderung nach begrifflicher Konsistenz hinausläuft. Dann unterscheidet sie sich nicht von der auf Entscheidungskonsistenz abzielenden Logik der Präjudizienbindung und der Systematizität der Rechtsdogmatik. Doch greift die Kritik zu kurz: Die Kontingenzformel impliziert sehr viel mehr als bloße interne Konsistenz des Entscheidens: Kontingenz heißt Anders-möglich-Sein und zugleich Von-etwas-anderem-Abhängigsein. Die Kontingenzformel operiert auf der Grenze des Rechts zu seiner äußeren Umwelt und zielt auf historische Variabilität der Gerechtigkeit und zugleich auf deren Umweltabhängigkeit. Der Ruf nach Gerechtigkeit - und dies ist der Kern der Kontingenzformel - fordert, Konsequenzen zu ziehen aus der Abhängigkeit des Rechts von seiner Ökologie, von seiner sozialen, menschlichen und natürlichen Umwelten. Damit kommen jenseits formaler Konsistenz materielle Orientierungspunkte ins Spiel. In der Definition „adäquate Komplexität des konsistenten Entscheidens" ist der entscheidende Aspekt die soziale Adäquanz in ihrer Relation zur internen Konsistenz. Die Intention der Gerechtigkeit richtet sich nicht auf die Maximierung dogmatischer Konsistenz, sondern darauf, auf äußerst

\footnotetext{
${ }^{26}$ Luhmann (Fn. 11), 469f.

${ }^{27}$ Luhmann (1993) (Fn. 2), 223.

28 Niklas Luhmann (1981) "Gerechtigkeit in den Rechtssystemen der modernen Gesellschaft", in: Niklas Luhmann (Hrsg.) Ausdifferenzierung des Rechts: Beiträge zur Rechtssoziologie und Rechtstheorie, Frankfurt: Suhrkamp, 374-418, 388ff.; Luhmann (1993) (Fn. 2), $225 f$.

${ }_{29}$ Zum Beispiel Josef Esser (1970) Vorverständnis und Methodenwahl in der Rechtsfindung: Rationalitätsgarantien der richterlichen Entscheidungspraxis, 2. Aufl. 1972. Frankfurt: Athenäum, 202ff.; Thomas Raiser (2007) Grundlagen der Rechtssoziologie, Tübingen: Mohr Siebeck, 139ff.; Klaus F. Röhl (2001) Allgemeine Rechtslehre: Ein Lehrbuch, 2. Auflage, Köln: Heymann, § 53.
} 
divergente Anforderungen von außen sensibel zu antworten und dabei möglichst hohe Konsistenz anzustreben. Die Kontingenzformel zielt nicht auf eine dem Recht immanente, sondern eine das Recht transzendierende Gerechtigkeit. Interne Konsistenz plus Responsivität gegenüber ökologischen Anforderungen - dies ist die Doppelformel juridische Gerechtigkeit. ${ }^{30}$

Im Gegensatz zu neukantianischen Theorien der Gerechtigkeit, welche die formalen und prozeduralen Anforderungen von Konsens und Universalisierung immer mehr verfeinern, konzentriert sich ein solches soziologisches Konzept auf die materiellen Aspekte der Beziehung des Rechts zu seiner Ökologie: Wird das Recht mit seinen Gleichheits-/Ungleichheitsprüfungen der heutigen polykontexturalen Gesellschaft gerecht? Wird es der natürlichen Umwelt gerecht? Wird es den Individuen gerecht? Die ökologische Orientierung des Rechts im weitesten Sinn dürfte der wichtigste Beitrag sein, den die Systemtheorie mit ihrer beharrlichen Betonung der System-Umwelt-Differenz in der Gerechtigkeitsdebatte erbringt. Gerechtigkeit lenkt die Aufmerksamkeit des positiven Rechts auf die problematische Frage seiner Adäquanz im Verhältnis zur Außenwelt.

Doch bedarf dies einer Qualifizierung. Denn gleichzeitig bringt die Systemtheorie, indem sie auf der selbstreferentiellen Schließung des Rechts insistiert, einen fundamentalen Widerspruch in der ökologischen Orientierung der Gerechtigkeit ans Licht. Die empathische Hetero-Referentialität des Rechts, wie sie im Sinne der Gerechtigkeit als Adäquanz des Rechts im Verhältnis zu Gesellschaft, Menschen und Natur erforderlich wäre, kann nicht durch ein Ausgreifen des Rechts in die Außenwelt erreicht werden, sondern ausschließlich innerhalb des Rechts. Es ist gefangen in den Verkettungen seiner selbstreferentiellen Operationen, die über Gleichheit/Ungleichheit von Einzelfällen befinden. In diesem Widerspruch liegt das Herzstück der Wirksamkeit von Gerechtigkeit in der heutigen Zeit: Wie ist Gerechtigkeit als eine Selbst-Transzendierung der Grenzen des Rechts möglich, wenn sie doch unausweichlich in der selbstreferentiellen Schließung des Rechtssystems gefangen ist? Gerechtigkeit als die notwendige, jedoch unmögliche Selbst-Transzendierung der rechtlichen Schließung - dies scheint einzig und allein denkbar als rechtliche coincidentia oppositorum.

Wie kann Gerechtigkeit die Schließung des Rechts transzendieren, wenn der Geltungstransfer auf der Basis des binären Codes Recht/Unrecht ausschließlich in den rekursiven Ketten von Gerichtsentscheidungen, gesetzgeberischen und vertraglichen Akten abläuft? Die Gerechtigkeit sieht sich hier mit der primären Schließung des Rechts konfrontiert: operative Geschlossenheit durch die Verkettung von Rechtsakten - Rechtsstrukturen - Rechtsakten. Aufgrund der unproduktiven Selbstbezüglichkeit und der radikalen Absonderung des Rechts von seiner sozialen Umwelt $^{31}$ ist die operative Geschlossenheit selbst bekanntlich zu einer bedeutenden

\footnotetext{
${ }^{30}$ Im einzelnen zu einem solchen im weitesten Sinne umweltbezogenen Gerechtigkeitsbegriff Teubner (Fn. 23), 123ff., besonders 147f.; ders. (Fn. 15), 218; Gunther Teubner und Peer Zumbansen (2000) "Rechtsverfremdungen: Zum gesellschaftlichen Mehrwert des zwölften Kamels", in: Gunther Teubner (Hrsg.) Die Rückgabe des zwölften Kamels: Niklas Luhmann in der Diskussion über Gerechtigkeit, Stuttgart: Lucius \& Lucius, 189-215; Gunther Teubner (2005) "Dreiers Luhmann", in: Robert Alexy (Hrsg.) Integratives Verstehen. Zur Rechtsphilosophie Ralf Dreiers, Tübingen: Mohr Siebeck, 199-211, 201ff.; ders. (Fn. 18), 185ff.

${ }^{31}$ Teubner (Fn. 23), 21ff.; Niklas Luhmann (1988) "Closure and Openness: On Reality in the World of Law", in: Gunther Teubner (Hrsg.) Autopoietic Law: A New Approach to Law and Society, Berlin: de Gruyter, 335-348; Luhmann (1993) (Fn. 2), 38ff.
} 
Quelle von Ungerechtigkeiten im Recht geworden. Mit guten Gründen verlangen deshalb kommunitaristische Kritiker des modernen Rechts radikale Veränderungen, um die Grenzen des positivierten Rechts aufzubrechen, um das formale Recht in die Gesellschaft zu reintegrieren und um alternative Foren, Prozeduren und Kriterien einer „communal justice“ zu etablieren. ${ }^{32}$ Doch wird man einräumen müssen, dass die Praktiken der Gerechtigkeit im modernen Recht eine andere Richtung eingeschlagen haben. Juridische Gerechtigkeit durchbricht die operative Geschlossenheit nicht, indem sie zur sozialen Einbettung der primären Operationen des Rechts zurückfindet. Vielmehr „transzendiert“ sie das Recht erst auf der Stufe seiner zweiten Schließung, auf der Ebene der rechtlichen Selbstbeobachtungen. ${ }^{33}$ Seit der entscheidenden Transformation des Rechts, als die juristische Argumentation bei Gerichtsverfahren, Gesetzgebung und Vertragsschlüssen damit anfing, Argumente ad hoc und ad hominem auszuschließen und darauf insistierte, nur noch auf spezialisierte rechtliche Materialien zu verweisen (Präjudizien, Regeln, Prinzipien), wurde der Gerechtigkeitsdiskurs zu dem Teil rechtlicher Selbstbeobachtungen, der den Blick auf die Grenzen des Rechts richtet und in der Beobachtung diese zu überschreiten versucht. Wann immer die Schließung rechtlicher Operationen durch die Schließung rechtlicher Selbstbeobachtungen komplettiert wurde, haben sich die Praktiken der Gerechtigkeit stets auf die Adäquanz des Rechts gegenüber seinen Umwelten konzentriert.

Aber wie sollte Gerechtigkeit als eine Selbstbeobachtungspraxis innerhalb des Rechts dazu imstande sein, die primäre Schließung des Rechts zu überwinden? Die Begründung lautet "re-entry" des Außerrechtlichen in das Rechtliche. Während rechtliche Operationen mit Hilfe ihrer Sequentialisierung eine Grenze zwischen Recht und Nicht-Recht, zwischen rechtlicher Kommunikation und anderen Arten sozialer Kommunikation, errichten, nutzen rechtliche Selbstbeobachtungen eben diese Unterscheidung „Recht/Nicht-Recht“, nun aber innerhalb der symbolischen Welt des Rechts. ${ }^{34}$ Immer wenn die Unterscheidung „Recht/Nicht-Recht“ („Nicht-Recht“ im Sinne von „außerrechtlich“, nicht „rechtswidrig“!) in die Sequenz rechtlicher Operationen wieder eintritt und die juristische Argumentation in die Lage versetzt wird, zwischen Normen und Tatsachen, zwischen internen Rechtsakten und externen Sozialakten, zwischen Rechtsbegriffen und gesellschaftlichen Interessen sowie zwischen internen Wirklichkeitskonstruktionen rechtlicher und sozialer Prozesse unterscheiden zu können, dann ist der Moment gekommen, in dem der Gerechtigkeitsdiskurs eine Entscheidung über diese Unterscheidungen verhandelt und die Frage aufwirft, ob die rechtlichen Entscheidungen denjenigen Aspekten der Außenwelt gerecht werden, wie sie intern rekonstruiert worden sind. ${ }^{35}$ Dies ist die paradoxe Leistung der doppelten Schließung - der Operationen und der

\footnotetext{
${ }^{32}$ Etwa Cotterrell (Fn. 1), 65ff.; 91ff., 315ff.; kritisch zur operativen Schließung des Rechts auch Michel van de Kerchove und François Ost (1992) Le droit ou les paradoxes du jeu, Paris: Presses Universitaires de France, $101 \mathrm{ff}$.

${ }^{33}$ Zur doppelten Schließung als notwendiger Bedingung der Selbstorganisation im allgemeinen Heinz von Foerster (1993) Wissen und Gewissen, Frankfurt: Suhrkamp, 25ff., 46ff.; in formalen Organisationen Niklas Luhmann (2000) Organisation und Entscheidung, Opladen: Westdeutscher Verlag, Kapitel 7.

${ }^{34}$ Luhmann (1993) (Fn. 2), 66ff.; 338ff.

35 Zum Umweltverhältnis von Organisationen Karl E. Weick (1985) Der Prozeß des Organisierens, Frankfurt: Suhrkamp; zu "enaction” als Alternative zu „representation“ Francisco J. Varela (1992) "Whence Perceptual Meaning? A Cartography of Current Ideas", in: F.J. Varela und J.P. Dupuy (Hrsg.) Understanding Origins: Contemporary Views on the Origin of Life, Mind and Society, Dordrecht: Kluwer, 235-263, 235ff.
} 
Beobachtungen. Während sowohl normproduzierende Rechtsakte und normverknüpfende Argumente in ihrem je geschlossenen Kreis interner Verkettungen verbleiben, setzt Gerechtigkeit als rechtliche Selbstbeobachtung von Recht/NichtRecht mit Hilfe der internen Unterscheidung von Selbstreferenz und Fremdreferenz das Recht zu seiner sozialen Umwelt (wohlgemerkt: „enacted environment, not real environment") ins Verhältnis und fragt nach seiner ökologischen Adäquanz.

Gerechtigkeit als diskursive Praxis zieht Konsequenzen aus dem re-entry des Außerrechtlichen in das Recht. Sie macht sich die epistemische Konfusion (à la Magritte: "This is not a pipe") über den Realitätsstatus der fremdreferentiellen Beobachtungen des Rechts zunutze. Resultat des re-entry ist der bereits erwähnte „imaginäre Raums“ innerhalb des Rechts, der sich aber selbst als Realität versteht. ${ }^{36}$ Bei ihrem Urteil über die ökologische Adäquanz des Rechts kann Gerechtigkeit gar nicht anders als nur mit Fiktionen über die Außenwelt zu arbeiten - doch sie muß sie als Realitäten behandeln. Deshalb kann Gerechtigkeit nur innerhalb dieses imaginären Raums im Recht operieren, der durch den re-entry der Ökologie in das Recht, durch die interne rechtliche Rekonstruktion externer Forderungen von Gesellschaft, Menschen und Natur, entsteht.

Die gesellschaftliche Abhängigkeit der Kontingenzformel "Gerechtigkeit" zeigt sich besonders in ihrer Affinität zu den großen historischen Prinzipien der sozialen Differenzierung. An dieser Stelle wird nun die Theorie der Gerechtigkeit ihrerseits abhängig - von den Subventionen der Gesellschaftstheorie. Wie schon eingangs erwähnt, sind die Kriterien der Gerechtigkeit nicht einfach geschichtlichem Wandel unterworfen, sondern ko-variieren mit Prinzipien sozialer Differenzierung. In einer stratifizierten Gesellschaft ist es natürliche und notwendige Voraussetzung der Gerechtigkeit, den sozialen Rang der streitenden Prozessparteien in vollem Umfang zu berücksichtigen. Justitia ist nicht blind! Die berühmte Formel des suum cuique aus heutiger Sicht eher eine Leerformel - macht für Menschen, die in legitimierten Hierarchien sozialer Stratifikation leben, konkreten Sinn. Wie Lawrence Rosen in seinen empirischen Studien zur Anthropologie der Gerechtigkeit gezeigt hat, trifft dies auf das traditionelle islamische Recht zu, dessen Gerechtigkeit verlangt, dass die soziale Stellung der Parteien und ihre Netzwerke im Prozess peinlichst genau rekonstruiert und in der Rechtsentscheidung explizit berücksichtigt werden. ${ }^{37}$ Max Weber irrte, als er dies abwertend „Kadi-Justiz" nannte, weil sie nach seiner Auffassung den grundlegenden Anforderungen universaler Gerechtigkeit nicht entsprach. ${ }^{38}$ Und auch in der alteuropäischen Gesellschaft war es natürlich und legitim, dass das Recht Adlige, die partes maiores gegenüber Stadtbevölkerung und Bauern privilegierte. Man stand schon an der Schwelle zur Moderne, als Michael Kohlhaas mit Gewalt gegen ein Recht protestierte, das adlige Pferdediebe ihm, dem gewöhnlichen Pferdehändler, gegenüber privilegierte. ${ }^{39}$ Während die justitia mediatrix des Mittelalters in einer vertikal-hierarchischen Weise zwischen göttlichem,

\footnotetext{
${ }^{36}$ George Spencer Brown (1997) Gesetze der Form, Lübeck: Bohmeier.

${ }^{37}$ Lawrence Rosen (1989) The Anthropology of Justice: Law as Culture in Islamic Society, Cambridge: Cambridge University Press, 58ff.

${ }^{38}$ Max Weber (1921) Wirtschaft und Gesellschaft., 2. vermehrte Aufl. 1925, 5. Aufl. 1976 Tübingen: Mohr \& Siebeck, 564ff.

${ }^{39}$ Heinrich von Kleist (1963) "Michael Kohlhaas", in: (Hrsg.) Kleists Werke Bd. 1, Weimar: Volksverlag, 81-187. Dazu die aufschlussreiche soziologische Interpretation von Michael Kauppert (2005) "Gesellschaftsstruktur und Gerechtigkeit in Heinrich von Kleists Michael Kohlhaas", in: Michael Corsten, Hartmut Rosa und Ralph Schrader (Hrsg.) Die Gerechtigkeit der Gesellschaft, Wiesbaden: Verlag für Sozialwissenschaften, 75-100.
} 
natürlichem und menschlichem Recht vermittelte, ${ }^{40}$ vermittelt die Gerechtigkeit der Moderne in einem horizontal-heterarchischen Modus zwischen der Eigennormativität des Rechts und der Eigennormativität seiner sozialen, menschlichen und natürlichen Umwelten. Heute sucht das Recht seine Gerechtigkeitskriterien, in seinen Umwelten, in verschiedenen gesellschaftlichen Diskursen, im pädagogischen, wissenschaftlichen, medizinischen, politischen oder wirtschaftlichen Diskurs, und verhilft ihnen in einem komplizierten Prozesses ihrer rechtlichen Rekonstruktion zur Rechtsgeltung. Trotz des Gleichheitsgebots der Verfassung rechtfertigt das Verfassungsrecht Ungleichbehandlungen, wenn diese entsprechend den pädagogischen, wissenschaftlichen, medizinischen, usw., d.h. „vernünftigen“ Maßstäben gerechtfertigt sind.

Ist dies ein neues Naturrecht, welches Gott, Natur und Vernunft durch die Prinzipien gesellschaftlicher Differenzierung ersetzt, also ein soziologisches Naturrecht? In der Sache unterläuft dieses Gerechtigkeitskonzept die Differenz von Positivismus und Naturrecht. Es erklärt beide für richtig und falsch zugleich. Mit dem Naturrecht teilt es den Impuls, Gerechtigkeit in einer von der Außenwelt des Rechts stammenden Orientierung zu suchen. Doch mit dem Positivismus hat es gemein, dass die Gerechtigkeit nicht von externen Autoritäten, weder von Gott, noch von der Natur, noch von der natürlichen Vernunft vorgegeben ist, sondern nur innerhalb des Rechts erarbeitet werden kann.

Die Wendung gegen das Naturrecht besteht darin, dass externe Autoritäten keine substantielle Gerechtigkeitskriterien bieten können. Die Wendung gegen den Positivismus aber besteht darin, dass Gerechtigkeit nicht von der bloßen Rekursion rechtlicher Dezisionen produziert werden kann. Das Gegenteil ist der Fall: Gerechtigkeit sabotiert Rechtsentscheidungen. Entgegen dem Drang des Rechts nach Entscheidungsgewissheit eröffnet juridische Gerechtigkeit als eine diskursive Praxis einen neuen Raum der Ungewissheit und der Indeterminiertheit des Rechts. Gerechtigkeit unterminiert die Routine der Rekursion rechtlicher Entscheidungen und fragt beharrlich, ob ein Streitfall im Lichte externer Anforderungen an das Recht nicht anders entschieden werden muss. Gerechtigkeit wirkt rechtsintern als eine subversive Kraft, mit der das Recht gegen sich selbst protestiert. Gerechtigkeit protestiert gegen die natürlichen Tendenzen des Rechts, sich an Präjudiz, Routine, Sicherheit, Stabilität, Autorität und Tradition zu binden. Gegen die dem Recht eingebauten Tendenzen wohlgeordneter Selbstkontinuierung spielt Gerechtigkeit ihre Präferenz für Unordnung, Revolte, Abweichung, Variabilität und Veränderung aus. Sie protestiert im Namen der Gesellschaft, der Menschen und der Natur - doch sie tut dies aus dem inneren Arkanum des Rechts. Subversive Gerechtigkeit ist der Stachel im Fleisch. Meuterei auf der Bounty - dies ist die Botschaft der Soziologie für die juridische Gerechtigkeit.

\section{Statt Rationalität: Das Irrationale in der Selbst- Transzendierung des Rechts}

Aber warum gerade Meuterei? Warum nicht Gerechtigkeit als Außenangriff aufs Recht im Namen der Gesellschaft? Dass Menschen, die ihre Hoffnungen ins Recht gesetzt haben, nach einem verlorenen Prozess das Recht für seine Ungerechtigkeit

\footnotetext{
${ }^{40}$ Placentinus (1192) "Quaestiones de iuris subtilitatibus", in: H. Fitting (1894) (Hrsg.) Quaestiones de iuris subtilitatibus des Irnerius, Berlin: J. Guttentag, 53.
} 
verantwortlich machen, ist zu erwarten. Aber dass der Widerstand aus dem inneren Arkanum des Rechts stammt - dies ist das Skandalon. Die Ursache für den Aufstand von innen, für die Selbstsubversion des Rechts im Namen der Gerechtigkeit, liegt in dem wohl folgenreichsten Versagen des Rechts: Es ist prinzipiell nicht in der Lage ist, sein eigenes Versprechen zu halten - das Versprechen, seine Entscheidungen auf eine einsichtige Basis vernünftiger Gründe zu stützen. Rechtliche Begründungen, wie professionell sie auch gearbeitet sind, können rechtliche Entscheidungen nicht rechtfertigen - jeder, der auch nur einmal einen Rechtsfall zu entscheiden hatte, hat diese ernüchternde Erfahrung gemacht. Anders ausgedrückt: Der wohl beunruhigendste Mangel des Rechts besteht darin, dass es das Eindringen von Irrationalität in die rationale Welt der normorientierten Entscheidungen und der vernunftbasierten Argumentation nicht verhindern kann. Deshalb waren Rechtspraktiker schon immer skeptisch gegenüber der Leistungsfähigkeit rationaler Gerechtigkeitstheorien nach der Machart von Rawls und Habermas. Die Gerechtigkeitsphilosophen sind sich ihrerseits des irrationalen Elements in rechtlichen Entscheidungen bewusst, doch sie versuchen es mit Exorzismus. Indem sie die Rolle des rationalen Arguments im Recht ad infinitum erweitern, um der Entscheidung Halt zu geben, treiben sie den Teufel der Paradoxien der Selbstreferenz aus. ${ }^{41}$ Natürlich vergeblich.

Im Gegensatz dazu stellen die provokativsten aktuellen Analysen des Rechtsversagens, wie sie von Jacques Derrida und Niklas Luhmann angefertigt wurden, die Aporien der Gerechtigkeit und die Entscheidungsparadoxien des Rechts gerade ins Zentrum ihrer Überlegungen. ${ }^{42}$ Wohlgemerkt, sie re-analysieren damit nur eine alte Erfahrung im Recht, das sich mit ehrwürdigen Selbstbeschreibungen in die berühmt-berüchtigten Doppelformeln geflüchtet hat - ratio et voluntas sowie ratio et auctoritas -, um mit den Grenzen der Vernunft in Rechtsentscheidungen leben zu können. ${ }^{43}$ Sogar die analytische Jurisprudenz, die anders als Dekonstruktion oder Autopoiesis nicht gerade unter dem Verdacht des Irrationalismus steht, muss die Grenzen des rationalen Argumentierens im Recht zur Kenntnis nehmen und zugeben, dass die logische Anwendung von Normen auf Sachverhalte nur dann funktioniert, wenn der Richter zusätzliche Prämissen in den Syllogismus einbringt. Sie muss ferner zugestehen, dass die Rechtfertigung von Normen durch innen zugrunde liegende Normen und Prinzipien unvermeidlich im Münchhausen-Trilemma endet: infiniter Regress, willkürlicher Abbruch oder Zirkularität. ${ }^{44}$ Die Unzulänglichkeit der Vernunft, Rechtsentscheidungen zu begründen, treiben die Critical Legal Studies in ihre Obsessionen der indeterminacy des Rechts. Sie treiben Carl Schmitt in die Obsessionen des Dezisionismus. Und es verwundert nicht, dass nun zahlreiche interdisziplinären Analysen einspringen, um die „Krankheit“ des Rechts mit ihren

\footnotetext{
${ }^{41}$ Typisch für diese Strategie Jürgen Habermas (1971) "Vorbereitende Bemerkungen zu einer Theorie der kommunikativen Kompetenz", in: Jürgen Habermas und Niklas Luhmann (Hrsg.) Theorie der Gesellschaft oder Sozialtechnologie - Was leistet die Systemforschung, Frankfurt: Suhrkamp, 101-141, 123ff., Jürgen Habermas (1973) "Wahrheitstheorien", in: Helmut Fahrenbach (Hrsg.) Wirklichkeit und Reflexion: Walter Schulz zum 60. Geburtstag, Pfullingen: Neske, 211-265, 255ff.

${ }^{42}$ Derrida (1991) (Fn. 2), 46ff.; Luhmann (1993) (Fn. 2), 307ff.

${ }^{43}$ Eine umsichtige Interpretation der Eumeniden des Aischylos unter dem Aspekt der Entscheidungsparadoxie des Rechts bietet Marie Theres Fögen (2007)

"Die Tragödie des Entscheidens: Eine Anmerkung zu den „Eumeniden“ des Aischylos", Ancilla Juris (anci.ch), 42-47.

${ }^{44}$ Hans Albert (1968) "Das Problem der Begründung", in: Hans Albert (Hrsg.) Traktat über kritische Vernunft, Tübingen: Mohr Siebeck, 9-34 und später ab 5. Auflage, UTB-Ausgabe 1991, die Anhänge I, insbesondere S. 220-242, Anhänge II und III.
} 
spezifischen Mitteln zu heilen: Psychologie mit dem affektiven Element, Psychoanalyse mit dem Unbewussten in Rechtsentscheidungen, Ökonomie mit Effizienzberechnungen, Soziologie mit Klassenstruktur oder sozialen Normen, Politikwissenschaft mit Policy-Erwägungen, sozialen Antagonismen oder reinem Voluntarismus der Macht, usw. usw. Doch wie reagiert das heutige Recht selbst auf seine fundamentale Unzulänglichkeit?

Juridische Gerechtigkeit ist als die Reaktion des Rechts auf sein eigenes Versagen zu verstehen. Sie kann, wie schon angedeutet, weder mit einem rechtsphilosophischen Konstrukt noch mit einem rechtlichen Entscheidungskriterium identifiziert werden, sondern erscheint als eine strukturierte soziale Dynamik innerhalb des Rechts. Juridische Gerechtigkeit wäre, dann, um die folgenden Überlegungen in einer Formel vorwegzunehmen, ein eigensinniger Prozess der Selbstbeschreibung im Recht, der die ungestörte Selbstreproduktion des Rechtssystems, die routinisierte Rekursivität der Rechtsoperationen, unterbricht, blockiert, sabotiert, unterminiert, der damit das Recht zu seiner Selbstranszendierung über jeden Sinn hinaus zwingt, der sich aber sogleich wieder unter den Fortsetzungszwang, weitere Rechtsoperationen zu produzieren, setzt und sich dadurch selbst sabotiert, dass er genau dadurch neue Ungerechtigkeiten schafft. Denn nach dem Durchgang durch die „irrationale“ Transzendenzerfahrung, nach dem "Gang durch die Wüste“, nötigt er sich dazu, diese Erfahrung unter den restriktiven Bedingungen des Rechtssystems - Entscheidungszwang, Normierungszwang, Begründungszwang - zu rekonstruieren. Unerbittliche Konsequenz dieses Fortsetzungszwangs ist es aber, dass der Rechtsprozess, auch und gerade nach der Selbst-Provokation in seiner Selbstbeschreibung "Gerechtigkeit", die sich in neuen Rechtskriterien übersetzt, immer neue Ungerechtigkeit erzeugt, gegen welche die Gerechtigkeit dann erneut protestieren muß, um sich dann wieder den Zwängen des Rechtssystems auszusetzen. Und so fort und so fort - in einer selbstquälerischen Daueroszillation.

Gerechtigkeit als diskursive Praxis ist also nicht einfach Subversion des positiven Rechts im Namen seiner Umwelten, sondern eine sich selbst steigernde zyklische Dynamik der Selbst-Subversion, in dem positive Rechtsentscheidungen durch rechtseigene Gerechtigkeitsproteste unterminiert werden und umgekehrt. Weder stellt diese Praxis Gerechtigkeit neu her, noch vervollkommnet sie eine bereits bestehende unvollkommene Gerechtigkeit, noch nähert sie sich einem Gerechtigkeitsideal asymptotisch an, vielmehr baut sie immer wieder aufs Neue die beiden Positionen auf - positive Rechtsentscheidung und Gerechtigkeitsprotest -, um sie beide sogleich wieder zu zerstören. Die Praxis verwirklicht und verunmöglicht Gerechtigkeit in einer ständigen Transformation von der Immanenz zur Transzendenz des Rechts und zurück zur Immanenz. Letztlich heizt sie damit nur die innere Unruhe, die Nervosität des Rechtsprozesses, die Daueroszillation zwischen beiden Polen, die notwendige Kontingenz des Rechts stets aufs Neue an.

Hervorzuheben ist, dass das Phänomen nicht einfach auf einen „dunklen Drang" nach Gerechtigkeit reduziert werden kann, der den rationalen Rechtsgang ständig stört und inn gelegentlich dazu anregt, besseres Recht zu produzieren. ${ }^{45}$ Stattdessen bezeichnet juridische Gerechtigkeit eine ganz spezifisch strukturierte,

\footnotetext{
${ }^{45}$ Analog zu Jürgen Habermas' Verständnis der Dauerirritation der institutionalisierten Politik durch Protestbewegungen, Jürgen Habermas (1985) "Die Neue Unübersichtlichkeit: Die Krise des Wohlfahrtsstaates und die Erschöpfung utopischer Energien", 39 Merkur, 1-14.
} 
theoretisch näher beschreibbare und empirisch identifizierbare Dynamik innerhalb des Rechtsgeschehens. Wohlgemerkt, mit Rechtsgeschehen ist hier nicht einfach der professionelle und organisierte Rechtsbetrieb, sondern jegliche ernstgemeinte Kommunikation über Recht, also gerade auch der Rechtsprotest der Bevölkerung „draußen im Lande“, gemeint. Derridisierend könnte man von „justiciance“ sprechen, um das Iterative, das ständige Changieren und Verschieben, die permanenten Bedeutungsveränderungen und das Nie-Abgeschlossene der Gerechtigkeit zu bezeichnen. Dies würde die auf das juridische Entscheidungsparadox konzentrierte Selbstsubversionsdynamik bezeichnen, die im Rechtsprozess stets mitlaufende Gerechtigkeitssuche, die aber im deutlichen Unterschied zur allgemeinen Sehnsucht nach Gerechtigkeit unter scharf einschränkenden Bedingungen steht. Und zwar ist die den Prozess leitende Suchformel durch eine merkwürdige Kombination von hoher Unbestimmheit und hoher Strukturiertheit ausgezeichnet. Bemerkenswert ist daran, dass das Verhältnis von Unbestimmtheit und Strukturiertheit nicht als Vermittlung, Kompromiß oder ein Treffen in der Mitte als "relativ strukturierte Unbestimmtheit" zu verstehen ist, sondern als eine wechselseitige Radikalisierung beider: „Chaos in Ordnung bringen" - die Doppelbedeutung dieser berühmten Formulierung Adornos macht die Radikalität der Gerechtigkeitsformel in der Moderne deutlich: den Rechtsgang zu chaotisieren, das Chaos in die Ordnung des Rechtsganges zu zwingen. ${ }^{46}$

Die Dynamik rechtsinterner Gerechtigkeit wird durch die vom Rechtssystem diktierten Ausgangsbedingungen ausgelöst und wird zugleich den vom Rechtssystem diktierten Beschränkungen ihrer Lösungsmöglichkeiten ausgesetzt. Damit ist der juridische Gerechtigkeit ihre gesellschaftsweite und ihre historische Universalisierung verboten. Sie bleibt system- und zeitgebunden. Die konkreten Beschränkungen en gros et en détail zu analysieren, ist die Aufgabe einer rechtssoziologischen Gerechtigkeitstheorie. Vorläufig lassen sich die Unterschiede einer juridischen Gerechtigkeitspraxis zum "dunklen Drang“ nach Gerechtigkeit folgendermaßen umreißen:

\section{Ausgangsbedingungen}

Die Besonderheiten dieser rechtssysteminternen Suchdynamik zeigen sich daran, dass sie nicht einfach vom undefinierbaren Begehren nach einer gerechten Welt angetrieben wird, sondern dass sie immer dann ausgelöst wird, wenn Rechtsverfahren und Rechtsargumentation auf den im Rechtsgang genauer lokalisierbaren Hiatus stoßen, der den laufenden Rechtsprozess unvermeidlich zum Stocken bringt. Systemtheoretisch gesprochen entsteht dieser Hiatus in der Verknüpfung Operation - Struktur - Operation (Rechtsakt - RechtsnormRechtsakt). Entgegen allen Vorurteilen gegenüber der Autopoiese als einer automatisch funktionierenden Mechanik $^{47}$ hat die Systemtheorie immer wieder deutlich gemacht, daß die Kette der Selbstreproduktion in jedem einzelnen Übergang von Struktur zu Operation (Erwartung zu Kommunikation) notwendig einen Bruch aufweist. Operationen erzeugen zwar Strukturen, Strukturen können aber nicht die darauffolgenden Operationen erzeugen, sondern können nur einen verdichteten

\footnotetext{
46 Wiethölter benutzt das Adorno-Zitat besonders gern im Zusammenhang von Rechtsentscheidungen, Wiethölter (1994) (Fn. 11), 107. Theodor Adorno (2003) Minima Moralia: Reflexionen aus dem beschädigten Leben, Frankfurt am Main: Suhrkamp, Teil 3, 1946/47, 103.

${ }^{47}$ Hubert Rottleuthner (1988) "Biological Metaphors in Legal Thought", in: Gunther Teubner (Hrsg.) Autopoietic Law: A New Approach to Law and Society, Berlin: De Gruyter, 97-127, 117.
} 
Möglichkeitsraum schaffen, in dem dann eine neue Operation "geschieht“. Diese muß aber stets, auch bei dichtester Struktur, ein Moment fundamentaler Ungewißheit überwinden. ${ }^{48}$ "Insofern entsteht mit Strukturbildung immer auch ein dazu nötiges Maß an Unsicherheit, und man wird nicht ohne Schadenfreude, gerade an sicherheitsfanatische Strukturbildungen wie Bürokratien und Rechtsordnungen feststellen können, wie mit der Zunahme der Bürokratisierung und der Verrechtlichung sich auch die Unsicherheit multipliziert. "“9 Auf das Recht gewendet: Jeder Rechtsakt (Gesetz, Vertrag, Richterentscheidung) verändert die Rechtslage, indem er eine neue Rechtsnorm erzeugt. Aber diese Rechtsnormen können ihrerseits keinen neuen Rechtsakt selbsttätig hervorbringen, sondern nur mehr oder weniger verdichtete Verweisungen auf mögliche neue Rechtsakte. ${ }^{50}$

Genau an dieser Stelle beginnt nun, um den Hiatus zu überspringen, die juristische Argumentation ihr rastloses Werk - erfolgreich und zugleich vergeblich. Juristische Argumentation bewirkt zwar Entscheidendes, aber auch sie kann den Konflikt nicht entscheiden, sie kann - entgegen den Selbstproklamationen von Argumentationstheoretikern - den Bruch von Struktur zu Operation, von Norm zu Rechtsakt nicht überbrücken. Juristisches Argumentieren kann nur Differenzen transformieren und stellt dann vor eine neue Entscheidungsalternative. Es verwandelt die bestehende Entscheidungsalternative in eine andere, die, wenn es gut geht, dem Rechtskonflikt adäquater ist als die begründungslose Alternative. Juristisches Argumentieren entscheidet nicht. Es rechtfertigt nicht. Es hat auch nichts zu verbergen. Es transformiert nur Entscheidungsalternativen, diese freilich drastisch. Eine Entscheidung bleibt nach wie vor notwendig, nur dass nach der Rechtsargumentation die sich präsentierende Entscheidungsalternative eine andere geworden ist. ${ }^{51}$

Welcher neue Rechtsakt dann schließlich angeschlossen wird, aber bleibt im Dunkel. An dieser Stelle, an der Differenz Struktur/Operation, Rechtsnorm/Rechtsakt oder Argumentation/Rechtsakt öffnet sich der Hiatus, der auch von rationalen Begründungen nicht überbrückbare Zwischenraum von Norm und Entscheidung. Die Aporien der juristischen Entscheidung sind selbst nicht rationalem Diskurs zugänglich, nicht begründbar, nicht rechtfertigbar, weder gerecht noch ungerecht. Wenn aber Gerechtigkeit gesucht werden soll, dann heißt dies, dass dieser Hiatus nicht dezisionistisch übersprungen wird oder durch ständige weitere inner- oder außerrechtliche Rationalisierungen bis zum Ermüdungspunkt immer nur verschoben wird. Der Gerechtigkeitsdiskurs wählt den Rejektionswert der Alternative von Dezisionismus oder Rationalisierung. Er macht die Aporie der Rechtsentscheidung (bekanntlich das Nichtweiterkommen beim Durchqueren des Flusses) durch einen reflexiven Akt der Selbstbeschreibung überhaupt erst bewusst, versucht also nicht, ihr auszuweichen oder sie einfach zu negieren, sondern artikuliert inn offen als Grenze der rationalen Begründbarkeit, bringt inn zur schmerzhaften Erfahrung und steigert inn ins Unerträgliche. ${ }^{52}$ Dieser Versuch, die Aporien des Rechtsganges durch Steigerung der Reflexivität bis hin zur Selbsttranszendierung des Rechts zu überwinden, ist die notwendige Ausgangsbedingung des Gerechtigkeitsprozesses im

\footnotetext{
${ }^{48}$ Niklas Luhmann (1984) Soziale Systeme: Grundriß einer allgemeinen Theorie, Frankfurt: Suhrkamp, $382 \mathrm{ff}$.

${ }^{49}$ Luhmann (1984) (Fn. 48), 391.

${ }^{50}$ Luhmann (1993) (Fn. 2), $49 f$.

${ }^{51}$ Teubner und Zumbansen (Fn. 30), 195 ff.; Luhmann (1993) (Fn. 2), 338ff.

${ }^{52}$ Derrida (1991) (Fn. 2), 46 ff.; Luhmann (1993) (Fn. 2), 307 ff.
} 
Inneren des Rechts, in dem nicht etwa die Gesellschaft, die Sozialtheorien oder eine andere externe Instanz dem Recht ihre normativen Maßstäbe diktierte, sondern in dem das Recht sich selbst den Prozess macht. ${ }^{53}$

\section{Selbsttranszendierung}

Die größten Schwierigkeit stellen sich freilich, wenn man zu verstehen sucht, was im Diskurs der Gerechtigkeit die Selbsttranszendierung des Rechts angesichts des Hiatus von Rechtsnorm und Rechtsakt bedeutet. Oben schon hatten wir mit Luhmann einen ersten Anlauf unternommen, um einen ökologischen Begriff der Gerechtigkeit zu profilieren, der aus der operativen Geschlossenheit des Rechts herausfindet und dennoch in ihr verharrt und hatten dies am Phänomen des „reentry" festgemacht. Die Kriterien der Gerechtigkeit werden dann nicht irgendwo außerhalb des Rechts aufgefunden, sondern das Recht kann sich nur in der Weise selbst transzendieren, daß es im selbsterzeugten Wiedereintritt diejenigen Umwelten, aus denen der Rechtskonflikt stammt - Gesellschaft, Natur, Mensch - von sich selbst unterscheidet, um dann im Verhältnis zu diesen „enacted ecologies“ umweltadäquate Gerechtigkeitskriterien zu etablieren. Damit ist von vornherein ausgeschlossen, dass das Recht solche Kriterien von der Außenwelt importieren kann, vielmehr muß es sie mit seinem eigenen Weltwissen selbsttätig konstruieren. Dieser re-entry in die Entscheidungspraxis des Rechts eben macht die Besonderheit einer juridischen Gerechtigkeit aus und ihren Unterschied zu rechtsexternen Vorstellungen einer gerechten Gesellschaft, einer politischen Kollektiventscheidungsgerechtigkeit oder einer moralisch-philosophischen Achtungsreziprozitätsgerechtigkeit. Die Suche kann ihre Kriterien nicht externalisieren, also weder auf Demokratie, noch auf Moral, noch auf Ökonomie hoffen, sondern ist auf sich selbst zurückgeworfen. Das Recht trägt die Verantwortung für seine Kriterien der Gerechtigkeit selbst.

Über eine solche ökologische Konzeption der Gerechtigkeit hinaus macht nun Derrida drei weitausgreifende Gedankenschritte, mit denen er der aktuellen Gerechtigkeitsdiskussion außerordentlich starke Impulse gegeben hat. Im ersten Schritt wagt er einen neuen Umgang mit dem Rechtsparadox. Luhmann identifiziert noch die Entscheidungsparadoxie des Rechtes, fordert dann aber mit Entschiedenheit, sie zu verstecken, zu verleugnen, zu verdrängen und schnellstens eine de-paradoxierende Unterscheidung einzuführen. Derrida dagegen verlangt, sich der Erfahrung der Paradoxie zu stellen und damit das juridische Denken in eine Welt zu treiben, in der Luhmann nur Paralyse und Schrecken vermutet. Gerechtigkeit wäre dann mehr als Konsistenzformel, aber auch mehr als Kontingenzformel, wäre Transzendenzformel, wäre „Anruf, Abgrund, Disruption, Widerspruchserfahrung, Chaos innerhalb des Rechts“. ${ }^{4}$ Das hat durchaus praktische Folgerungen für

\footnotetext{
${ }^{53}$ Rudolf Wiethölter (2003) "Recht-Fertigungen eines Gesellschafts-Rechts", in: Christian Joerges und Gunther Teubner (Hrsg.) Rechtsverfassungsrecht: Rechtfertigung zwischen Privatrechtsdogmatik und Gesellschaftstheorie, Baden-Baden: Nomos, 1-45, 19f., ein Text des Autors, der sich als Selbstüberbietung früherer Texte lesen lässt, etwa von Rudolf Wiethölter (1989) "Ist unserem Recht der Prozeß zu machen?" in: Axel Honneth, Thomas McCarthy, Claus Offe und Albrecht Wellmer (Hrsg.) Zwischenbetrachtungen: Im Prozeß der Aufklärung. Jürgen Habermas zum 60. Geburtstag, Frankfurt: Suhrkamp, 794-812.

${ }^{54}$ Derrida (1991) (Fn. 2). Dazu aufschlußreich Fatima Barijij-Kastner (2007) Ohnmachtssemantiken: Systemtheorie und Dekonstruktion, Weilerswist: Velbrück, besonders Kap. III.2.
} 
Rechtserkenntnis und -entscheidung: Veränderung der Situation als Entscheidung sub specie aeternitatis und nicht nur sub specie societatis. ${ }^{55}$

Im zweiten Schritt radikalisiert Derrida, was Selbsttranszendierung des Rechts überhaupt bedeuten kann. Luhmann verlangt in der Gerechtigkeit vom Recht eine Selbsttranszendierung in Richtung seiner selbstentworfenen Umwelten, macht dort aber auch halt. Damit resigniert er letztlich angesichts der Defizienzen des re-entry. Wenn re-entry immer nur Rekonstruktion des Außen im Innen, immer nur „enactment" einer Umwelt ist, dann ist er immer zugleich Einschließung und Ausschließung. Was aber vom Recht ausgeschlossen ist, fordert dennoch Einlaß in die Gerechtigkeit. Das Perturbationsereignis selbst, die Verwirrungen und Erschütterungen, die es auslöst, bleiben in Luhmanns Analysen seltsam unterbelichtet. Hier produziert die Systemtheorie mit ihrer Leitentscheidung System/Umwelt ihren eigenen blinden Fleck, der ihr nicht erlaubt, das „Zwischen“ im Perturbationsgeschehen näher zu analysieren. ${ }^{56}$ Luhmann sieht nur und kann nur sehen, was innerhalb der Grenzen des Rechts passiert und widmet seine Aufmerksamkeit nur den nach der Perturbation getroffenen Unterscheidungen. Derrida dagegen fordert das Überschreiten auch dieser Grenze und mutet dem Gerechtigkeitsdiskurs zu, sich den Verwirrungen und Erschütterungen auszusetzen, die Transzendenz jenseits jeden Sinnes - „den Gang durch die Wüste“ - zu erfahren. Und dies ist in der Tat für den heutigen Wissenschaftsstil befremdlich: ein Transzendieren jeden Sinnes, ein Verweis auf mystische Gewalt, eine Begegnung mit dem Anderen der Levinasschen Alteritätsphilosphie, eine Herausforderung der modernen Rationalitäten durch "reine" Gerechtigkeit, Gabe, Freundschaft, Verzeihung.

Im dritten Schritt schließlich verändert sich das Verhältnis von religiöser zu einer spezifisch juridischen Transzendenz. Während Luhmann die Transzendenzerfahrung auf das Religionssystem konzentriert und damit implizit andere Teilsysteme, unter anderen das Rechtssystem, ausschließt, ist Derridas dekonstruktives Denken darauf gerichtet, das Transzendenzbewußtsein aus seiner modernen Isolierung in der Religion herauszulösen und in die hochrationalisierten Welten der Wirtschaft, der Wissenschaft, der Politik und des Rechts wieder einzubringen. Derrida zieht damit nur die Konsequenz aus dem - auch und gerade Luhmann zugänglichen - Phänomen, dass trotz aller gesellschaftlichen Arbeitsteilung Wissen sich nicht auf Wissenschaft konzentrieren läßt, dass Machtprozesse trotz des staatlichen Gewaltmonopols auch außerhalb der Politik stattfinden, dass die Unterscheidung Recht/Unrecht trotz aller Formalisierung des Rechtssystems auch außerhalb des Rechts praktiziert wird.

Entsprechend lassen sich auch Transzendenzerfahrungen, auf die sich die Energien der Religion konzentrieren, nicht auf die Religion begrenzen, sondern erzeugen auch und gerade in den hochspezialisierten Sinnwelten der Moderne Wirkungen, die sich von denen religiöser Transzendenz deutlich unterscheiden. Daß

\footnotetext{
${ }^{55}$ An dieser Stelle wäre der Unterschied zu Fögen (Fn. 43), 47 zu markieren, die das Entscheidungsparadox mit der Feststellung enden lässt:

„Ein letzter Trost heisst: Und es funktioniert trotzdem, das Entscheiden, tagtäglich, bei Gericht und and erswo"

${ }^{56}$ Zum blinden Fleck der Systemtheorie und zu Möglichkeiten, inn auszuleuchten Gunther Teubner (1997) "Im blinden Fleck der Systeme: Die Hybridisierung des Vertrages", 3 Soziale Systeme, 313326.
} 
Max Weber die seltsam anmutende Formulierung eines „Polytheismus“ der Moderne benutzt hat, gewinnt eigentlich erst in diesem je eigenen Transzendenzbezug jeder hochgetriebenen Teilrationalität seine eigentümliche Bedeutung, die verloren geht, wenn man inn auf eine bloße Rationalitätenvielfalt oder einen Polyzentrismus reduziert. Es geht gerade um die Vielfalt möglicher Transzendenzzugänge, so könnte man Max Weber lesen. Denn dies war schließlich eine der Leistungen des alten Polytheismus, aus Differenzen der Transzendenz Differenzen in der Immanenz, insbesondere soziale Rollen, Kompetenzen und Funktionen, zu legitimieren.

Man sollte Derrida so lesen: Er stellt hier die erstaunliche These auf, dass jede moderne Institution ihre spezifische Selbsttranszendierung kennt, die je unterschiedliche paradoxale Wirkungen hat: er nennt dies die Wirkung der „reinen Gabe“ gegenüber der profitgesteuerten Wirtschaft, der „Freundschaft" gegenüber der professionalisierten Politik, der „Verzeihung“ gegenüber der säkularisierten Moral und vor allem der „Gerechtigkeit" gegenüber dem hochtechnisierten Recht. ${ }^{57}$ Sie alle sind der jeweiligen Eigenlogik der Institutionen entstammende Verweisungsüberschüsse, die utopische Energien in ganz anderen Räumen als dem der Religion reaktivieren.

Denkt man dies für das Recht weiter, so lässt sich Gerechtigkeit als eine Transzendenzerfahrung verstehen, die gerade nicht mit religiöser Transzendenz identisch ist. Worin aber besteht ihre Eigentümlichkeit? Die Antwort könnte sich daraus ergeben, dass der Gerechtigkeitsdiskurs dort beginnt, wo das Recht endet am Hiatus zwischen Norm und Entscheidung, wo das Rechtsparadox auftaucht. Der Gerechtigkeitsdiskurs schließt gerade an die verwirrende Erfahrung des spezifischen Rechtsparadoxes an und überschreitet eben an dieser Stelle die Sinngrenzen des Rechts. Ist es Recht, die Unterscheidung Recht/Unrecht auf die Welt anzuwenden ${ }^{58}$ Aber genau das ist die Gerechtigkeitsfrage. Sobald das Recht auf sein eigenes Paradox stößt, dann ist es mit Notwendigkeit der Gerechtigkeitsfrage ausgesetzt! Und nicht der Frage der Freigebigkeit, der Freundschaft, der Verzeihung oder gar des Seelenheils. In der Selbsttranszendierung des Rechts bleibt also die Besonderheit des Rechtsparadoxes Recht/Unrecht wirksam. Sie ist als die spezifische Grenzüberschreitung des Rechts notwendig, aber gerade deswegen nicht mehr in der rationalen Sprache des Rechts ausdrückbar, sondern nur noch in verrätselter Sprache, irrealer Idealisierung, Gleichnis, Symbolisierung, Literatur, Traum, Delirium, Utopie. ${ }^{59}$

Eine solche Gerechtigkeitssuche kann nicht darauf hoffen, im Recht selbst oder in der Gesellschaft oder gar in der Religion Kriterien der Gerechtigkeit zu finden, sondern muß über jeglichen Sinn hinaus das Recht überschreiten, die Erfahrung der spezifischen Transzendenz des Rechts machen, unter deren Eindruck sie wieder

\footnotetext{
57 Jacques Derrida (1993) Falschgeld: Zeitgeben I, München: Fink, besonders49ff.; Jacques Derrida (2000) Politik der Freundschaft, Frankfurt: Suhrkamp; Jacques Derrida (2000) "Jahrhundert der Vergebung. Möglichkeiten und Grenzen des Verzeihens", 48 Lettre International, 10-18; Derrida (1991) (Fn. 2), besonders 44ff.

${ }^{58}$ Mit Luhmann und gegen Luhmann verbietet sich Rainer Maria Kiesow, die im Rechtsparadox verborgene Gerechtigkeitsfrage zu stellen, um dann aber das Problem (zwar nicht mehr „rechtlich“, sondern) „politisch“, wiederkehren zu lassen: „politisch wird es immer wiederkehren“, Rainer Maria Kiesow (2004) "Error iudicis: Fünf Gänge und ein Rätsel", in: Rainer Maria Kiesow und Henning Schmidgen (Hrsg.) Das Irrsal hilft, Berlin: Merve, 29-45, 39, 44. Mit dem „Politischen“ dürfte Kiesow allerdings weniger die institutionalisierte Politik, sondern das helfende Irrsal selbst meinen.

59 Dies macht die Unverständlichkeit der Sprache verständlich, mit deren Hilfe Benjamin, Derrida oder Wiethölter ihr Denken über das Recht verrätseln. Und alle Versuche, sie zu enträtseln, vergeblich.
} 
zurück in die Immanenz des Rechts muß, um sub specie aeternitatis Recht zu sprechen. Was aber ist von Gerechtigkeit als expliziter Transzendenzerfahrung zu halten, wenn Nietzsche recht hat: „Gott ist tot"? Kann man in der säkularisierten Gesellschaft die Transzendenz des Rechts ohne Religion „denken“? Wäre das nicht ein Naturrecht ohne Gott, aber auch ohne Vernunft? Und schließlich: Hat dann die bisher rätselhafteste Bestimmung von Gerechtigkeit, die sich bei Johannes findet, noch irgend einen Sinn: „Um die Gerechtigkeit aber, dass ich zum Vater gehe, und Ihr mich fort nicht sehet"? ${ }^{60}$

Verlangt wäre, die Gerechtigkeit in einer Situation neuzuinterpretieren, in der Transzendenz nur noch ohne Gott gedacht werden kann, aber dennoch gedacht werden muß. Genau an dieser Stelle setzen Levinas und Derrida ein mit ihrer positiven Bestimmung einer „philosophischen Transzendenz“, die der Totalität des Sinnes die Exteriorität der Transzendenz gegenüberstellt, in der die Gerechtigkeit als unendliche Anforderung des einen Anderen und die der vielen Anderen aufscheint. ${ }^{61}$ Allerdings muß man hier der Radikalität des Anderen im Denken von Levinas und Derrida gerecht werden. Mit Alterität ist nicht einfach das ethische Prinzip der Fürsorge für den anderen oder die Berücksichtigung der Singularität der Individualperspektive gemeint, ${ }^{62}$ sondern die gerade nicht-sprachlich vermittelte, nicht-phänomenologische Erfahrung des anderen, eine Transzendenzerfahrung im „Antlitz des Anderen“. Gegenüber dem Bestehen auf Begründbarkeit der Gerechtigkeit, auf der Rationalität der öffentlichen Rede bestünde man auf der Gerechtigkeit des Nichtbegründbaren, dem nicht-rationalem Anderen der Gerechtigkeit. Gerechtigkeit wäre dann angesiedelt an der Grenze von Immanenz des Rechts zu seiner Transzendenz. Gerechtigkeit letztlich als die Suche, den Bruch der Immanenz mit der Transzendenz zu überwinden - „Hingang zum Vater“ -, das bedeutete die Aufforderung der Transzendenz, die Immanenz in deren für diese jedoch nicht verstehbaren Sinne zu transformieren. Gerechtigkeit ist dann kein Maßstab in „tadelloser Idealität“, sondern ein „Prozess der Verwandlung des Unrechts in Recht". ${ }^{63}$

Johannes' eigentliche Botschaft geht jedoch darüber noch hinaus. Die Inkarnation wird der Transzendenz selbst noch nicht gerecht, sondern erst die Erfahrung fundamentaler Ungerechtigkeit macht Gerechtigkeit möglich. Gerechtigkeit verwirklicht sich erst im realen Durchgang durch Ungerechtigkeit, Leiden und Schmerz, Gerechtigkeit ist Transformation des Leidens, mehr noch, ist Selbst-Opfer, das erst die Trennung Immanenz/Transzendenz aufheben kann. In diesem Sinne bedeutet „Hingang zum Vater" Aufhebung der Trennung Immanenz/Transzendenz durch die Transformation von Ungerechtigkeit. Das Leiden ist auch und gerade durch die Suche nach Gerechtigkeit selbst ausgelöst. Also ein Leiden an der Gerechtigkeit als der vergeblichen Suche und deren ungerechten Konsequenzen, weil sie in der Immanenz des Rechts - in der "Notordnung" - „verwirklicht" werden muß. Was Johannes der Debatte hinzufügt, wäre also, dass Gerechtigkeit als ein

\footnotetext{
${ }^{60}$ Joh., 16, 10. Dazu die subtile Interpretation von Horst Folkers (2000) "Johannes mit Aristoteles ins Gespräch über die Gerechtigkeit vertieft: Epilegomena zum 12. Kamel des Niklas Luhmann", 21 Zeitschrift für Rechtssoziologie, 61-107, besonders 68ff.

${ }^{61}$ Emmanuel Levinas (1992) Jenseits des Seins oder anders als Sein geschieht, Freiburg: Alber, 342ff.; Emmanuel Levinas (1987) Totalität und Unendlichkeit: Versuch über die Exteriorität, Freiburg: Alber, $125 \mathrm{ff}$.

${ }^{62}$ So aber Axel Honneth (2000) Das Andere der Gerechtigkeit: Aufsätze zur praktischen Philosophie, Frankfurt am Main: Suhrkamp, 154ff., 165ff.

${ }^{63}$ Folkers (2000) (Fn. 60), 71 f.
} 
Transformationsprozess des Rechts verstanden werden muß, der überhaupt erst durch die reale Erfahrung von Ungerechtigkeit möglich wird. Dieser Gedanke findet heute sein schwaches Echo in Durkheim's colère publique, die in der Normverletzung die Ursache für die Normbildung findet und in Gerechtigkeitstheorien, die den „sense of injustice" als Auslöser von Normierungsprozessen betrachten. ${ }^{64}$

„Unsichtbar für die Menschen“? Das bedeutet nicht nur Unzugänglichkeit der Transzendenz, sondern „ein letztes Freigestelltsein des Rechts eines jeden einzelnen von den endlichen Bedingungen jeder Art menschlicher Rechtsgewährung". ${ }^{65}$ Doch nur in einer Welt mit Gott könnte die Erlösungshoffnung durch Gerechtigkeit bestehen. Wenn aber Transzendenz ohne Gott gedacht wird, dann ist keine Erlösung in der Gerechtigkeit möglich. Was dann bleibt, ist nur noch der verzweifelte Suchprozeß selbst, der die permanente innere Unruhe des Rechts erzeugt, der sich auf seiner Suche immer wieder anderen Erfahrungen der Ungerechtigkeit aussetzt, der ruhelos neue Rechtskriterien der Gleichheit konstruiert und ständig neue Entscheidungsbegründungen erfindet und gerade dadurch aufs Neue die Gerechtigkeit zerstört. Die Suche nach Gerechtigkeit wird zu einer bloßen Sucht des Rechts, zerstörerisch und erfinderisch zugleich.

\section{Anschlußzwänge}

Die Unterschiede einer spezifisch juridischen Gerechtigkeit gegenüber dem "dunklen Drang" werden besonders deutlich, wenn man die drastischen Einschränkungen bedenkt, zu denen das moderne Rechtssystem seine eigene Kontingenzformulierung nach dem Delirium ihrer Selbsttranszendierung zwingt. Juridische Gerechtigkeit kann nicht im Zugriff aufs Ganze die Ungerechtigkeit des Rechts mit der der Welt identifizieren. Sie ist genötigt, genau an der oben beschriebenen nicht-negierbaren Defizienz der Rechtsentscheidung anzusetzen, um dann ihrerseits die fehlende Verbindung zwischen Struktur und Operation, zwischen Rechtsnorm und Entscheidung, nicht nur bewußt zu machen, sondern auch diese mit eigenen Mitteln irgendwie, wie unbefriedigend auch immer, tatsächlich herzustellen. Genau an dieser Stelle unterscheidet sich die juridische Gerechtigkeit vom „Rechtspietismus" des radikalen Flügels der Freirechtsschule, welche „die Gleichbehandlung des Gleichen und somit jede Möglichkeit der Verallgemeinerung konkreter, typisierenden Pflichten zu allgemein formulierenden Sollenssätzen“ ablehnte. $^{66}$ Denn hier setzt der spezifische Anschlusszwang im Rechtssystem die unendliche Gerechtigkeit drei unterschiedlich wirkenden drastischen Beschränkungen aus.

Sie muß die Struktur/Entscheidung-Verknüpfung innerhalb des durch den Code Recht/Unrecht und deren Programme scharf limitierten Möglichkeitsraum der Rechtsentscheidung herstellen, selbst dann wenn es ihrer eigenen Überzeugung widerspricht - Entscheidungszwang. ${ }^{67}$ Selbst wenn der Richter nach einem langen

\footnotetext{
${ }^{64}$ Émile Durkheim (1992) Über soziale Arbeitsteilung: Studie über die Organisation höherer Gesellschaften, Frankfurt: Suhrkamp, 118ff.; Edmond N. Cahn (1949) The Sense of Injustice, New York: New York University Press.

${ }^{65}$ Folkers (Fn. 60), 76 f.

${ }^{66}$ Franz Wieacker (1967) Privatrechtsgeschichte der Neuzeit unter besonderer Berücksichtigung der deutschen Entwicklung, 2. Aufl. Göttingen: Vandenhoeck \& Ruprecht, 580 und Fußnote 57.

67 Luhmann (1993) (Fn. 2), 307ff.; Marie Theres Fögen (2004) "Schrittmacher des Rechts: Anmerkungen zum Justiz- und Rechtsverweigerungsverbot", in: Heinrich Honsell (Hrsg.) Privatrecht und Methode: Festschrift für Ernst A. Kramer, Basel: Helbing und Lichtenhahn, 3-20.
} 
und quälenden Prozess des Nachdenkens und Diskutierens weiß, dass beide Parteien des Rechtsprozesses „Recht haben“ und selbst wenn er weiß, dass er, wie er auch entscheidet, einer Partei Unrecht tut, muß er der Klage stattgeben oder die Klage abweisen - unter der Anforderung der Gerechtigkeit. ${ }^{68}$ Tertium non datur.

Zugleich setzt das Rechtssystem der Gerechtigkeitssuche kaum zu verkraftende kognitive Zwänge. Der Suche ist es nicht erlaubt, sich im irrationalen Gefühl der Ungerechtigkeit oder im vagen Sehnen nach Gerechtigkeit zu verlieren, sondern sie wird durch die oben genannten spezifisch strukturierten Aporien des Rechtsgangs gezwungen, sich zwar mit aller Intensität der Erfahrung der Irrationalität, des Rechtsgefühls, der Alterität, des Leidens, des Schmerzes, der Leere und der Fülle der Transzendenz auszusetzen, sie muß diese Erfahrung dann aber in rationale Gründe, rechtstechnische Argumente, anschlussfähige Rechtsdogmatik transformieren - Begründungszwang. Hier kommt dann die von Luhmann so eindringlich beschriebene Schwierigkeit zum Ausdruck, mit rationalen Argumenten den hochgetriebenen Anforderungen der Außenwelt an das Recht responsiv entgegenzukommen, aber zugleich den internen Anforderungen an konsistente Fallentscheidung zu genügen.

Endgültig wird der Spielraum juridischer Gerechtigkeit dadurch eingeengt, dass sie nicht über alle Macht- und Einflußmittel dieser Welt verfügt, um eine gerechte Gesellschaft herstellen zu können, sondern nur über die im Vergleich hochspezialisierten zugleich und armseligen Operationen und Strukturen des Rechts, Rechtsakte und Rechtsnormen. Sie steht bei ihren Lösungsmöglichkeiten vor harten Einschränkungen, die sie vom weisen Willkürakt eines Machtsouveräns, vom dunklen Orakelspruch, von mystischer Offenbarung unterscheidet. Sie muß ihre überwältigenden Erfahrungen der Alterität, also die Erfahrungen der inneren Unendlichkeit des einzelnen Menschen, die Erfahrungen der Eigenrationalität und Eigennormativität der unterschiedlichen Diskurse, und die Erfahrungen der Irrationalität in einer geradezu absurd simplifizierenden Form zusammenziehen, der Formulierung einer Norm, die den Anspruch erhebt, dem Rechtskonflikt adäquat zu sein - Normierungszwang. ${ }^{69}$

Man kann die Wirkungen kaum überschätzen, die von diesen drei Zwängen auf die juridische Gerechtigkeit ausgehen. Entscheidungszwang: sie kann den Konflikt nicht in der Schwebe lassen, eine Partei muß Recht haben, die andere Unrecht; Begründungszwang: die Entscheidung muß auf Begründungen gestützt werden, die den untauglichen Versuch unternehmen, Konsistenz und Responsivität plausibel verbinden. Normierungszwang: die Entscheidung verlangt die Reduktion der komplexen Fallproblematik auf eine viel zu einfache Fallnorm. Unter solchen unzumutbaren Bedingen sollen dann neuartige Strukturen geschaffen werden, die den Hiatus überwinden und den „Sprung“ ermöglichen? In der Tat, vor den unendlichen Anforderungen der Gerechtigkeit wird eine recht bescheidene „Notordnung“ errichtet.

\footnotetext{
${ }^{68}$ So das berühmte Paradox des Rabbi, wiedererzählt von Henri Atlan, À tort et à raison: Intercritique de la science et du mythe (1986) S.? erste Seite des Textes. 1ff. Oder das berühmte Paradox der Athene in den Eumeniden des Aischylos, zeitgemäß interpretiert von Fögen (Fn. 43).

${ }^{69}$ Wolfgang Fikentscher (1977) Methoden des Rechts in vergleichender Darstellung, Bd.IV. Tübingen: Mohr \& Siebeck, insbesondere Kapitel 31-33.
} 
Wollen aber Gerechtigkeitstheorien von diesen Zwängen absehen - und dies sind nicht die wenigsten und durchweg die sensibelsten - diskreditieren sie sich selbst. Sie nehmen die Gerechtigkeit als radikale Transzendierung des Rechts ernst, aber sie unterschlagen die gegenläufigen Ansprüche der Transzendenz des Rechts, im Namen der Allgegenwärtigkeit die Gerechtigkeit in der Immanenz des Rechts zu verwirklichen. ${ }^{70}$ Sie exkludieren sich aus dem juridischen Gerechtigkeitsdiskurs, der seine Teilnehmer dazu zwingt, die Selbsttranszendierung des Rechts zu vollziehen, zugleich aber sie in juristische Normen, Argumente und Entscheidungen zurückzuübersetzen. Theorien, die sich diesem Zwang entziehen, mögen als philosophische Gerechtigkeitstheorien weiter wirken, sie mögen sogar als Stachel im Fleisch dem Recht große Schmerzen bereiten. Aber der Stachelschmerz lässt nach. Nach einer gewissen Zeit wird er gar nicht mehr registriert. Dieses Schicksal steigender Irrelevanz erleiden besonders „kritische“ Theorien des Rechts. Sie scheitern am ehernen Gesetz des Rechtsgangs: Kritik ohne Gegenvorschlag zählt nicht. ${ }^{71}$ Gerade ein „juristischer Negativismus“ kann sich selbst nur als Übergangserscheinung definieren, irgendwann muß und wird er die Bedingungen formulieren, unter denen Rechtsverbote in Freiheiten unter Auflagen erlassen werden sollen. $^{72}$ Die Anschlusszwänge des Rechts stellen nun einmal die Gerechtigkeitssuche vor eine andere Alternative, als es Adorno für die Moralphilosophie formulieren und entsprechend seine Präferenzen äußern konnte: für „konkrete Denunziation des Unmenschlichen“ und gegen „unverbindliche und abstrakte Situierung etwa des Seins des Menschen" ${ }^{73}$ Die temperamentvolle Rechtskritik der Critical Legal Studies wäre eine rechtssoziologisch-empirische Fallstudie wert, um der Selbstmarginalisierung hochbegabter und hochmotivierter Rechtsverweigerungsjuristen nachzugehen. Und gegenüber dem juridischen „Hic Rhodus" kann auch ein (links- oder rechts-)heideggerianischer Attentismus nicht bestehen, sei es Giorgios Agambens Hoffnungen auf eine neue Gemeinschaft, sei es Philip Nonets geduldiges Warten auf ..... ${ }^{74}$ Sowohl Luhmann als auch Derrida haben dies, jeder auf seine Weise, in aller Deutlichkeit ausgesprochen. Derrida geht in seiner Benjamin-Kritik sogar so weit, einer Gerechtigkeitstheorie, die sich weigert, in die Immanenz der Rechtskalkulationen zurückzukehren und sich stattdessen mit der Unterscheidung mythischer und mystischer Gewalt, deren Kriterien den Menschen unzugänglich bleiben müssten, zufriedengibt, Komplizität mit dem Schlimmsten vorzuwerfen. ${ }^{75}$

Jedoch sollte man darüber nicht die positiven Aspekte des juridischen Disziplinierungszwangs vergessen, die den Rechtsverweigerungsgerechtigkeitstheoretikern zu denken geben könnten: Er setzt das Recht unter einen außerordentlichen Innovationsdruck. Vor dem Doppel-

\footnotetext{
${ }^{70}$ So in aller Deutlichkeit Folkers (2000) (Fn. 60), 78.

${ }^{71}$ Nur scheinbar argumentiert Roellecke dagegen: Gerd Roellecke, "Kritik ohne Ersatzvorschlag ist noch lange kein Gedöns", Frankfurter Allgemeine Zeitung am 10.02.2006.

${ }^{72}$ Zum juristischen Negativismus Rudolf Wiethölter (1969) "Recht und Politik. Bemerkungen zu Peter Schwerdtners Kritik", Zeitschrift für Rechtspolitik, 155-158, 158 und zu Freiheiten unter Auflagen Wiethölter (2004) (Fn. 53), $20 f$.

${ }^{73}$ Adorno (1996) (Fn. 12) 261.

${ }^{74}$ Giorgio Agamben (2002) Homo Sacer: Die souveräne Macht und das nackte Leben, Frankfurt: Suhrkamp, 197f.; Giorgio Agamben (2004) Ausnahmezustand (Homo Sacer II.I), Frankfurt: Suhrkamp, 83ff.; Philippe Nonet (2007) "Time and Law", Theoretical Inquiries in Law, 311-332, 322 ff.

${ }^{75}$ Derrida (1991) (Fn. 2), 124 f. Heftige Kritik an einer solchen „erschreckenden Benjamin-Exegese“ und an der daraus resultierenden „bejahenden Dekonstruktion“ Derridas, die nicht mehr auf frontale Vernichtung, sondern auf eine „zirkuläre Entgrenzung des Rechts“ zielt, äußert Cornelia Vismann (1992) "Das Gesetz 'DER Dekonstruktion"', 11 Rechtshistorisches Journal, 250-264, 262.
} 
Imperativ „Chaos in Ordnung bringen“ kann keine Gesetzesnorm, kein Richterakt, kein Dogmatikkonstrukt bestehen, alles wird auf den Prüfstand der Gerechtigkeit gestellt. Ungleich schwieriger aber ist es, die gleichzeitig erhobene Forderung zu erfüllen, Ersatzvorschläge zu formulieren. Juridische Gerechtigkeit muß stets neue Gesetzesnormen, Richterakte, Rechtsbegriffe erfinden, die dem Anspruch standhalten, gerechter zu sein als die alten als ungerecht empfundenen Formeln. Damit wird eine komparative Dimension in das Recht eingeführt, die es erlaubt, ja die dazu nötigt, zwischen höheren und niederen Graden an Gerechtigkeit zu unterscheiden. Gerechter als andere wäre dann eine Rechtsordnung, die radikaler als andere ihre Selbsttranszendierung in unterschiedliche Dimensionen der Alterität zulässt und befördert, die aber dann auch Entscheidungen, Argumente und Normen produziert, die sich vor anderen Rechtsordnungen als gerechter erweisen lassen. Innovationsdruck heißt aber auch Innovationschance. Was wir oben als merkwürdige Eigenheit der juridischen Gerechtigkeitsformel angesprochen hatten, nämlich die Kombination von hoher Unbestimmtheit (Selbsttranszendierung) und hoher Bestimmtheit (Formzwang des Juridischen), begünstigt die Entfaltung kreativer Energien. Im „imaginären Raum“ des re-entry finden juridische Konstruktionsphantasien ihre große Chance. Nicht von ungefähr gelten die juristische Person, der konsensuelle Vertrag und das Konstrukt des Staates als zivilisatorische Errungenschaften ersten Ranges für Recht und Gesellschaft. Und die immer wieder neu erzählte Fabel vom „zwölften Kamel“ verweist auf geheime Gemeinsamkeiten von artistischer und juridischer Kreativität. ${ }^{76}$

\section{Effets pervers}

Doch das Merkwürdige ist: Die Rechtsverweigerer unter den Gerechtigkeitssuchern haben eigentlich recht. Um der Gerechtigkeit willen sind sie nicht bereit, den Preis für die dreifache Einschränkung des Gerechtigkeitsdiskurses zu zahlen. Denn der Preis dafür, die unendliche Gerechtigkeitserfahrung auf eine binär kodierte Entscheidung, auf deren responsiv-konsistente Begründung und deren konditionale Normierung zu reduzieren, ist hoch - neue Ungerechtigkeit. Besonders wegen der Armseligkeit des rechtlichen Formzwangs, aber auch wegen der mangelnden Sensibilität philosophischer Universalisierung schafft die juridische Gerechtigkeitssuche notwendig neue Ungerechtigkeit, die ihrerseits zu erneuter Selbsttranszendierung und $\mathrm{zu}$ erneuter Disziplinierung führt. Levinas: „Die allgemeinen und großzügigen Prinzipien können sich in der Anwendung verkehren. Jedes großzügige Denken wird von seinem eigenen Stalinismus bedroht. "77 Hier wird der Unterschied dieser zyklischen Sicht, in der Gerechtigkeit sich selbst unterminiert, zu einer hierarchischen Sicht der Gerechtigkeit, die durch auf rationale Gründe gestützte Entscheidungen eine Steigerung der Gerechtigkeit erhoffen, besonders fühlbar. Denn sie führt unnachsichtig vor Augen, dass gerade die Angewiesenheit

\footnotetext{
${ }^{76}$ Dazu die Beiträge in Gunther Teubner (Hrsg.) (2000) Die Rückgabe des zwölften Kamels: Niklas Luhmann in der Diskussion über Gerechtigkeit, Stuttgart: Lucius \& Lucius.

${ }^{77}$ Emmanuel Levinas (1982) L'au delà du verset, Paris: Les éditions de Minuit, 98. Hier liegt der Einsatzpunkt für Levinas' "Skeptizismus“ gegenüber philosophischen und juristischen Rationalisierungen der Gerechtigkeit, von deren Notwendigkeit er dennoch überzeugt ist, Levinas (1992) (Fn. 61), 364. Dazu aufschlussreich Christian Schlüter (2000) Gleichheit - Freiheit Gerechtigkeit: Versuch einer Ortsbestimmung in praktischer Absicht, abrufbar unter: http://dochost.rz.hu-berlin.de/dissertationen/schlueter-christian-2000-07-12/PDF/Schlueter.pdf, 196ff.; Hans-Dieter Gondek (1994) "Gesetz, Gerechtigkeit und Verantwortung bei Levinas", in: Anselm Haverkamp (Hrsg.) Gewalt und Gerechtigkeit: Derrida - Benjamin, Frankfurt: Suhrkamp, 315-330.
} 
des Rechts auf rationale Entscheidungen, Begründungen und Normierungen einer der vertracktesten der Ursprünge der Ungerechtigkeit unter den Menschen ist.

Die dunkelste Seite der juridischen Gerechtigkeit aber ist ihr unbändiger Drang zur Universalisierung ihrer selbst, ist die naheliegende Versuchung der „Vergerechtlichung“, dass sie ihre zweiseitige Logik - Selbsttranszendierung des Rechts und deren rechtliche Re-Disziplinierung - auf die ganze Gesellschaft ausdehnt. ${ }^{78}$ Statt sich im Gegensatz zu den ganz anderen Anforderungen politischer Verteilungsgerechtigkeit oder moralischer Achtungsgerechtigkeit auf Konfliktbewältigung zu beschränken, sucht sie im „akuten Gerechtigkeitsfieber“79 eine gerechte Gesellschaft mit den Mitteln juridischer Gerechtigkeit zu verwirklichen. Es ist gerecht, die Probleme der Welt nach dem binären Code Recht/Unrecht zu entscheiden - das ist das summum ius, summa iniuria der funktional differenzierten Gesellschaft. Sie teilt diesen Drang mit anderen Kontingenzformeln, mit dem der Wirtschaft, die ganze Welt als ein mit wirtschaftlichen Mitteln zu lösenden Knappheitsproblem zu beschreiben, mit dem der Legitimitätsformel der Politik und mit dem der Limitationalität der Wissenschaft. Sie alle versprechen, eine gute Gesellschaft mit ihren Mitteln herstellen zu können, obwohl sie doch nur äußerst begrenzte Teilantworten für ihren Bereich geben können. „Vergerechtlichung“ der Gesellschaft als Versuch, die gesamte Gesellschaft mit Rechtsinstrumentarien zur Gerechtigkeit zu bringen, ist einer der unheilvollen Rationalitätenimperialismen der Moderne, der Ökonomisierung, Politisierung, Verwissenschaftlichung, Moralisierung, Medikalisierung der Gesellschaft - ein unidirektionaler Steigerungsprozess der juridischen Gerechtigkeit, gegen den politische Wachsamkeit Not tut. Dieser Imperialismus einer nur partiellen Rationalität ist deshalb so gefährlich, weil er dem Verlangen der Menschen nach einer unteilbaren Gerechtigkeit auch in der heutigen Gesellschaft freundlich entgegenkommt, obwohl er weiß, dass dies Verlangen in der Moderne prinzipiell nicht gestillt werden kann, und dennoch dem mit juridischer Gerechtigkeit, die sich zur gesamtgesellschaftlichen Gerechtigkeit aufschwingt, ein falsches Heilsversprechen gibt. Beide erzeugen ein gefährliches Gebräu aus unbeantwortbaren Fragen und verlogenen Antworten. „Human Rights als das Ideal einer gerechten Gesellschaft" - dieser heute sich verbreitende Imperialismus einer entfesselten juridischen Gerechtigkeit produziert den totalitären Gerechtigkeitssucher der Moderne, der in seinen Rechtsausschweifungen die begrenzte Gerechtigkeit des Rechts auf die ganze Gesellschaft projiziert: „einen der rechtschaffensten zugleich und entsetzlichsten Menschen unserer Zeit" ${ }^{80}$

\section{Literatur}

Adorno, Theodor (2003) Minima Moralia: Reflexionen aus dem beschädigten Leben, Frankfurt am Main: Suhrkamp.

Adorno, Theodor W. (1973) Negative Dialektik, Frankfurt am Main: Suhrkamp.

- (1996) Nachgelassene Schriften Bd. 10: Probleme der Moralphilosophie, Frankfurt am Main: Suhrkamp.

Agamben, Giorgio (2002) Homo Sacer: Die souveräne Macht und das nackte Leben, Frankfurt: Suhrkamp.

- (2004) Ausnahmezustand (Homo Sacer II.I), Frankfurt: Suhrkamp.

\footnotetext{
${ }^{78}$ Dazu bemerkenswert Bernhard Schlink (2004) "Der Preis der Gerechtigkeit", 58 Merkur, 983-997.

${ }^{79}$ So der Arzt Relling über den gerechtigkeitssüchtigen Greger in Henrik Ibsen (1994, Orig. 1884), Die Wildente. Stuttgart, 67.

${ }^{80}$ Kleist (Fn. 39), 81; dazu die anregende Interpretation von Regina Ogorek (1988) "Adam Müllers Gegensatzphilosophie und die Rechtsausschweifungen des Michael Kohlhaas", 96-131,121ff.
} 
Albert, Hans (1968) "Das Problem der Begründung", in: Hans Albert (Hrsg.): Traktat über kritische Vernunft. Tübingen: Mohr Siebeck, 9-34.

Barjiji-Kastner, Fatima (2007) Ohnmachtssemantiken: Systemtheorie und Dekonstruktion, Weilerswist: Velbrück.

Buckel, Sonja (2007) Subjektivierung und Kohäsion: Zur Rekonstruktion einer materialistischen Theorie des Rechts, Weilerswist: Velbrück.

Cahn, Edmond N. (1949) The Sense of Injustice, New York: New York University Press.

Cotterell, Roger (2006) Law's Community: Legal Theory in Sociological Perspective, Oxford: Clarendon.

Derrida, Jacques (1991) Gesetzeskraft: Der 'mystische Grund der Autorität', Frankfurt: Suhrkamp.

- (1993) Falschgeld: Zeitgeben I, München: Fink.

- (1995) Marx' Gespenster: Der verschuldete Staat, die Trauerarbeit und die neue Internationale, Frankfurt: Fischer.

- (1999) Préjugés: Vor dem Gesetz, Wien: Passagen.

- (2000) "Jahrhundert der Vergebung. Möglichkeiten und Grenzen des Verzeihens", 48 Lettre International, 10-18.

- (2000) Politik der Freundschaft, Frankfurt: Suhrkamp.

Dreier, Ralf (2002) "Niklas Luhmanns Rechtsbegriff", 88 Archiv für Rechts- und

Sozialphilosophie, 305-322.

Durkheim, Emile (1977) Über die Teilung der sozialen Arbeit, Frankfurt: Suhrkamp.

Durkheim, Émile (1992) Über soziale Arbeitsteilung: Studie über die Organisation

höherer Gesellschaften, Frankfurt: Suhrkamp.

Esser, Josef (1970) Vorverständnis und Methodenwahl in der Rechtsfindung:

Rationalitätsgarantien der richterlichen Entscheidungspraxis, 2. Aufl. 1972. Frankfurt:

Athenäum.

Fikentscher, Wolfgang (1977) Methoden des Rechts in vergleichender Darstellung,

Bd.IV. Tübingen: Mohr \& Siebeck.

Foerster, Heinz von (1993) Wissen und Gewissen, Frankfurt: Suhrkamp.

Fögen, Marie Theres (2004) "Schrittmacher des Rechts: Anmerkungen zum Justizund Rechtsverweigerungsverbot", in: Heinrich Honsell (Hrsg.): Privatrecht und

Methode: Festschrift für Ernst A. Kramer. Basel: Helbing und Lichtenhahn, 3-20.

- (2007) "Die Tragödie des Entscheidens:

Eine Anmerkung zu den „Eumeniden“ des Aischylos", Ancilla Juris (anci.ch), 42-47.

Folkers, Horst (2000) "Johannes mit Aristoteles ins Gespräch über die Gerechtigkeit

vertieft: Epilegomena zum 12. Kamel des Niklas Luhmann", 21 Zeitschrift für

Rechtssoziologie, 61-107.

Forst, Rainer (1994) Kontexte der Gerechtigkeit: Politische Philosophie jenseits von Liberalismus und Kommunitarismus, Frankfurt: Suhrkamp.

- (1999) "Die Rechtfertigung der Gerechtigkeit. Rawls' Politischer Liberalismus und Habermas' Diskurstheorie in der Diskussion", in: Hauke Brunkhorst und Peter Niesen (Hrsg.): Das Recht der Republik. Festschrift für Ingeborg Maus. Frankfurt: Suhrkamp, 105-168.

Foucault, Michel (1976) Überwachen und Strafen: Die Geburt des Gefängnisses,

Frankfurt: Suhrkamp.

- (2002) Die Ordnung der Dinge, Frankfurt am Main: Suhrkamp.

Gondek, Hans-Dieter (1994) "Gesetz, Gerechtigkeit und Verantwortung bei Levinas", in: Anselm Haverkamp (Hrsg.): Gewalt und Gerechtigkeit: Derrida - Benjamin.

Frankfurt: Suhrkamp, 315-330.

Goodman, Nelson (1990) Weisen der Welterzeugung, Frankfurt: Suhrkamp. 
Günther, Gotthard (1976) "Cybernetic Ontology and Transjunctional Operations", in: Gotthard Günther (Hrsg.): Beiträge zur Grundlegung einer operationsfähigen Dialektik I. Hamburg: Meiner, 249-283.

- (1976) "Life as Poly-Contexturality", in: Gotthard Günther (Hrsg.): Beiträge zur Grundlegung einer operationsfähigen Dialektik I. Hamburg: Meiner, 283-306. Habermas, Jürgen (1971) "Vorbereitende Bemerkungen zu einer Theorie der kommunikativen Kompetenz", in: Jürgen Habermas und Niklas Luhmann (Hrsg.): Theorie der Gesellschaft oder Sozialtechnologie - Was leistet die Systemforschung. Frankfurt: Suhrkamp, 101-141.

- (1973) "Wahrheitstheorien", in: Helmut Fahrenbach (Hrsg.): Wirklichkeit und Reflexion: Walter Schulz zum 60. Geburtstag. Pfullingen: Neske, 211-265.

- (1985) "Die Neue Unübersichtlichkeit: Die Krise des Wohlfahrtsstaates und die Erschöpfung utopischer Energien", 39 Merkur, 1-14.

- (1992) Faktizität und Geltung, Frankfurt: Suhrkamp.

Honneth, Axel (2000) Das Andere der Gerechtigkeit: Aufsätze zur praktischen

Philosophie, Frankfurt am Main: Suhrkamp.

Kauppert, Michael (2005) "Gesellschaftsstruktur und Gerechtigkeit in Heinrich von Kleists Michael Kohlhaas", in: Michael Corsten, Hartmut Rosa und Ralph Schrader (Hrsg.): Die Gerechtigkeit der Gesellschaft. Wiesbaden: Verlag für Sozialwissenschaften, 75-100.

Kelsen, Hans (1960) "Das Problem der Gerechtigkeit", in: Hans Kelsen (Hrsg.):

Reine Rechtslehre. 2. Aufl. Wien: Denticke, 335-444.

Kerchove, Michel van de und François Ost (1992) Le droit ou les paradoxes du jeu, Paris: Presses Universitaires de France.

Kiesow, Rainer Maria (2004) "Error iudicis: Fünf Gänge und ein Rätsel", in: Rainer Maria Kiesow und Henning Schmidgen (Hrsg.): Das Irrsal hilft. Berlin: Merve, 29-45. Kleist, Heinrich von (1963) "Michael Kohlhaas", in: (Hrsg.): Kleists Werke Bd. 1. Weimar: Volksverlag, 81-187.

Koselleck, Reinhart (2006) "Begriffsgeschichtliche Probleme der Verfassungsgeschichtssschreibung", in: Reinhart Koselleck (Hrsg.):

Begriffsgeschichten: Studien zur Semantk und Pragmatik der politischen und sozialen Sprache. Frankfurt: Suhrkamp, 365-401.

Ladeur, Karl-Heinz (1992) Postmoderne Rechtstheorie: Selbstreferenz -

Selbstorganisation - Prozeduralisierung, Berlin: Duncker \& Humblot.

Levinas, Emmanuel (1982) L'au delà du verset, Paris: Les éditions de Minuit.

- (1987) Totalität und Unendlichkeit: Versuch über die Exteriorität, Freiburg: Alber.

- (1992) Jenseits des Seins oder anders als Sein geschieht, Freiburg: Alber.

Luhmann, Niklas (1974) Rechtssystem und Rechtsdogmatik, Stuttgart: Kohlhammer.

Luhmann, Niklas (1981) "Gerechtigkeit in den Rechtssystemen der modernen Gesellschaft", in: Niklas Luhmann (Hrsg.): Ausdifferenzierung des Rechts: Beiträge zur Rechtssoziologie und Rechtstheorie. Frankfurt: Suhrkamp, 374-418.

Luhmann, Niklas (1981) "Subjektive Rechte: Zum Umbau des Rechtsbewußtseins für die moderne Gesellschaft", in: Niklas Luhmann (Hrsg.): Gesellschaftsstruktur und Semantik Bd. 2. Frankfurt: Suhrkamp, 45-104.

- (1984) Soziale Systeme: Grundriß einer allgemeinen Theorie, Frankfurt: Suhrkamp. - (1988) "Closure and Openness: On Reality in the World of Law", in: Gunther Teubner (Hrsg.): Autopoietic Law: A New Approach to Law and Society. Berlin: de Gruyter, 335-348.

Luhmann, Niklas (1993) Das Recht der Gesellschaft, Frankfurt am Main: Suhrkamp. Luhmann, Niklas (1997) Die Gesellschaft der Gesellschaft, Frankfurt: Suhrkamp. - (2000) Organisation und Entscheidung, Opladen: Westdeutscher Verlag. 
Lyotard, Jean-François (1987) Der Widerstreit, 2. Aufl. 1989. München: Fink. Menke, Christoph (2004) Spiegelungen der Gleichheit, Frankfurt am Main: Suhrkamp.

Nonet, Philippe (2007) "Time and Law", Theoretical Inquiries in Law, 311-332. Ogorek, Regina (1988) "Adam Müllers Gegensatzphilosophie und die Rechtsausschweifungen des Michael Kohlhaas", 96-131.

Pauer-Studer, Herlinde (2000) Autonom leben: Reflexionen über Gleichheit und Freiheit, Frankfurt am Main: Suhrkamp.

Petersen, Hanne und Henrik Zahle (1995) Legal Polycentricity: Consequences of Pluralism in Law, Aldershot: Dartmouth.

Placentinus (1192) "Quaestiones de iuris subtilitatibus", in: H. Fitting (1894) (Hrsg.): Quaestiones de iuris subtilitatibus des Irnerius. Berlin: J. Guttentag, Raiser, Thomas (2007) Grundlagen der Rechtssoziologie, Tübingen: Mohr Siebeck. Rawls, John (1971) A Theory of Justice, Cambridge, Mass.: Harvard University Press.

- (1975) Eine Theorie der Gerechtigkeit, Frankfurt: Suhrkamp.

Author, "Kritik ohne Ersatzvorschlag ist noch lange kein Gedöns", Frankfurter Allgemeine Zeitung am 10.02.2006.

Röhl, Klaus F. (2001) Allgemeine Rechtslehre: Ein Lehrbuch, 2. Auflage, Köln: Heymann.

Rosen, Lawrence (1989) The Anthropology of Justice: Law as Culture in Islamic Society, Cambridge: Cambridge University Press.

Rottleuthner, Hubert (1988) "Biological Metaphors in Legal Thought", in: Gunther Teubner (Hrsg.): Autopoietic Law: A New Approach to Law and Society. Berlin: De Gruyter, 97-127.

Schlink, Bernhard (2004) "Der Preis der Gerechtigkeit", 58 Merkur, 983-997. Schlüter, Christian (2000) Gleichheit - Freiheit - Gerechtigkeit: Versuch einer Ortsbestimmung in praktischer Absicht, abrufbar unter: http://dochost.rz.huberlin.de/dissertationen/schlueter-christian-2000-07-12/PDF/Schlueter.pdf.

Spencer Brown, George (1997) Gesetze der Form, Lübeck: Bohmeier.

Teubner, Gunther (1989) Recht als autopoietisches System, Frankfurt: Suhrkamp.

- (1996) "Altera Pars Audiatur. Das Recht in der Kollision anderer Universalitätsansprüche", 65 Archiv für Rechts- und Sozialphilosophie. Beiheft, 199220.

- (1997) "Im blinden Fleck der Systeme: Die Hybridisierung des Vertrages", 3 Soziale Systeme, 313-326.

- (Hrsg.) (2000) Die Rückgabe des zwölften Kamels: Niklas Luhmann in der Diskussion über Gerechtigkeit, Stuttgart: Lucius \& Lucius.

- (2003) "Der Umgang mit Rechtsparadoxien: Derrida, Luhmann, Wiethölter", in: Christian Joerges und Gunther Teubner (Hrsg.): Rechtsverfassungsrecht: RechtFertigungen zwischen Sozialtheorie und Privatrechtsdogmatik. Baden-Baden: Nomos, 25-45.

- (2005) "Dreiers Luhmann", in: Robert Alexy (Hrsg.): Integratives Verstehen. Zur Rechtsphilosophie Ralf Dreiers. Tübingen: Mohr Siebeck, 199-211.

- (2006) "Die anonyme Matrix: Menschenrechtsverletzungen durch „private“ transnationale Akteure", 45 Der Staat, 161-187.

Teubner, Gunther und Peer Zumbansen (2000) "Rechtsverfremdungen: Zum gesellschaftlichen Mehrwert des zwölften Kamels", in: Gunther Teubner (Hrsg.): Die Rückgabe des zwölften Kamels: Niklas Luhmann in der Diskussion über Gerechtigkeit. Stuttgart: Lucius \& Lucius, 189-215. 
Varela, Francisco J. (1992) "Whence Perceptual Meaning? A Cartography of Current Ideas", in: F.J. Varela und J.P. Dupuy (Hrsg.): Understanding Origins: Contemporary Views on the Origin of Life, Mind and Society. Dordrecht: Kluwer, 235-263.

Vismann, Cornelia (1992) "Das Gesetz 'DER Dekonstruktion'", 11 Rechtshistorisches Journal, 250-264.

Walzer, Michael (1992) Sphären der Gerechtigkeit: Ein Plädoyer für Pluralität und Gleichheit, Frankfurt: Suhrkamp.

Weber, Max (1921) Wirtschaft und Gesellschaft., 2. vermehrte Aufl. 1925, 5. Aufl. 1976 Tübingen: Mohr \& Siebeck.

- (1968) Gesammelte Aufsätze zur Wissenschaftslehre, Tübingen: Mohr \& Siebeck. Weick, Karl E. (1985) Der Prozeß des Organisierens, Frankfurt: Suhrkamp.

Wieacker, Franz (1967) Privatrechtsgeschichte der Neuzeit unter besonderer Berücksichtigung der deutschen Entwicklung, 2. Aufl. Göttingen: Vandenhoeck \& Ruprecht.

Wiethölter, Rudolf (1969) "Recht und Politik. Bemerkungen zu Peter Schwerdtners Kritik", Zeitschrift für Rechtspolitik, 155-158.

- (1989) "Ist unserem Recht der Prozeß zu machen?" in: Axel Honneth, Thomas McCarthy, Claus Offe und Albrecht Wellmer (Hrsg.): Zwischenbetrachtungen: Im Prozeß der Aufklärung. Jürgen Habermas zum 60. Geburtstag. Frankfurt: Suhrkamp, 794-812.

- (1994) "Zur Argumentation im Recht: Entscheidungsfolgen als Rechtsgründe?" in: Gunther Teubner (Hrsg.): Entscheidungsfolgen als Rechtsgründe: Folgenorientiertes Argumentieren in rechtsvergleichender Sicht. Baden-Baden: Nomos, 89-120.

- (2003) "Recht-Fertigungen eines Gesellschafts-Rechts", in: Christian Joerges und Gunther Teubner (Hrsg.): Rechtsverfassungsrecht: Rechtfertigung zwischen

Privatrechtsdogmatik und Gesellschaftstheorie. Baden-Baden: Nomos, 1-45. Wittgenstein, Ludwig (1989) "Philosophische Untersuchungen", in: Ludwig Wittgenstein (Hrsg.): Werkausgabe Bd.1. Frankfurt: Suhrkamp, 234-580. 